\title{
Superconducting Order Parameter Symmetry in Multi-layer Cuprates
}

\author{
J. Maly, D. Z. Liu and K. Levin \\ Department of Physics, University of Chicago, Chicago, Illinois, 60637
}

(Submitted to Physical Review B on August 24, 1995)

\begin{abstract}
In this paper we classify the allowed order parameter symmetries in multilayer cuprates and discuss their physical consequences, with emphasis on Josephson tunneling and impurity scattering. Our solutions to the gap equation are based on highly non-specific forms for the inter- and intra-plane pairing interactions in order to arrive at the most general conclusions. Within this framework, the bi-layer $(N=2)$ case is discussed in detail with reference to YBCO and BSCCO and the related Landau-Ginzburg free energy functional. Particular attention is paid to the role of small orthorhombic distortions as would derive from the chains in YBCO and from superlattice effects in BSCCO, which give rise to a rich and complex behavior of the multilayer order parameter. This order parameter has $N$ components associated with each of the $N$ bands or layers. Moreover, these components have specific phase relationships. In the orthorhombic bi-layer case the $(s,-s)$ state is of special interest, since for a wide range of phase space, this state exhibits $\pi$ phase shifts in corner Josephson junction experiments. In addition, its transition temperature is found to be insensitive to non-magnetic inter-plane disorder, as would be present at the rare earth site in YBCO, for example. Of particular interest, also, are the role of van Hove singularities which are seen to stabilize states with $d_{x^{2}-y^{2}}$-like symmetry, (as well as nodeless $s$-states) and to elongate the gap functions along the four van Hove points, thereby leading to a substantial region of gaplessness. We find that $d_{x^{2}-y^{2}}$-like states are general solutions for repulsive interactions; they possess the fewest number of nodes and therefore the highest transition temperatures. In this way, they should not be specifically associated with a spin fluctuation driven pairing mechanism.
\end{abstract}

PACS numbers: 74.72.-h, 74.20.Mn, 74.50.+r, 74.62.-c 


\section{INTRODUCTION}

The question of the order parameter symmetry in layered cuprate superconductors is one of the most important issues currently under debate. Despite strong evidence for a $d_{x^{2}-y^{2}}$ state, at least in one particular cuprate (YBCO), there are still experimental inconsistencies.2. This matter is complicated further by the complexities of YBCO associated with the double copper-oxide plane structure. The same bi-layer unit is shared with the bismuth 2212 compounds (BSCCO), where recent photoemission data, 105 seem also to favor a $d_{x^{2}-y^{2}}$ symmetry. In the former compound, 6 if not in the latter, there is evidence that the double layer unit leads to two bands each of which crosses the Fermi surface and each of which presumably has a distinct superconducting order parameter. It is not a priori clear whether these two gaps have the same or opposite phase, nor whether they are predominantly associated with the same or different irreducible representations of the tetragonal symmetry group. It is, therefore, essential to provide a systematic classification of the order parameter in these bi-layer (and more general $N$-layer) systems before meaningful and unambiguous inferences can be deduced from the experimental data.

It is the purpose of this paper to expand upon earlier work by ourselves 8 and other groups 13 by classifying and establishing physical consequences of the various order parameter symmetries in $N$-layer systems. Of particular interest are those parameter sets which lead to $\pi$ junction behavior $4-17$ in an $a, b$-plane Josephson configuration. It is widely assumed that the observation of these $\pi$ phase shifts provides the strongest evidence yet for the $d_{x^{2}-y^{2}}$ state in YBCO. By contrast, we find that in the more general orthorhombic bi-layer case, in part because of van Hove effects, $\pi$ phase shifts are fairly widespread and not uniquely associated with the $d_{x^{2}-y^{2}}$ state. Moreover, the proximity to the van Hove singularities leads to a stabilization of the $d_{x^{2}-y^{2}}$ symmetry for a variety of different models for the pairing interaction, beyond the simple spin fluctuation model 18 It also is associated with a considerable distortion of the gap function away from the ideal representation of the $d_{x^{2}-y^{2}}$ state. In order to understand the physical consequences of various order parameter sets we investigate the role of intra- and inter-layer impurities, include a discussion of Josephson coupling, and present a more general analysis of our results in the context of both a Landau-Ginzburg theory and the solution of the arbitrary $N$-layer problem.

A number of authors have considered the possibility of electron tunneling and pair interactions of electrons on different planes in the context of a bilayer structure. Bulut and Scalapino 11 used numerical solutions of a strong coupling model to show that when both interlayer interactions and hopping were included in a bilayer model two competing states arose, one with $d_{x^{2}-y^{2}}$ solutions in phase on both sub-bands of the Fermi-surface and the other with $s$-states of opposite sign. Similar observations were made by Liechtenstein and co-workers $\theta$ who argued that the more probable situation for spin fluctuation induced superconductivity, corresponded to a pair of out-of-phase $s$ states. It should be noted that throughout this paper we will use the generic notation " $s$ " ( and " $d "$ ) as applying to gap functions which have the same (or different) signs under a rotation of the wave-vector by $\pi / 2$. It is important to be particularly clear on our notational convention since we emphacize the role of orthorhombicity in our work. Among other things, this orthorhombicity leads to what we refer to as "s- $d$ " or "mixed states", (although the overall sign under a $\pi / 2$ rotation remains either + or - , depending on which component is dominant). The possibility 
of mixed $s$ - $d$-states has been discussed within Landau-Ginzburg theory, in a mono-layer 19 as well as a bi-layer context.20 In the latter, a spontaneous $s$ - $d$ mixing was considered, using microscopic arguments based on an RVB decoupling scheme. While most bi-layer studies considered tetragonal symmetry, earlier work by our own group, $\mathrm{f}$ using the more conventional BCS pairing formulation, investigated the role of a small amount of $a, b$-axis anisotropy in the bi-layer problem. This small orthorhombicity appears to be amplified by van Hove effects. As a consequence, the solution corresponding to a pair of out of phase $s$-wave states frequently has the interesting and important physical consequence of leading to $\pi$ phase shifts in a corner Josephson tunneling experiment $14-17$ It should be stressed that orthorhombicity is assumed to enter via the chains in YBCO. There are alternate scenarios for this material which explicitly build in the chain bands 223 and their contribution to the superconducting gap. Here we ignore these explicit effects, in large part because recent tunneling studies indicate that the order parameter behavior is largely unaffected by reducing the oxygen stoichiometry to the limit where the chains are strongly fragmented 15 (and thus presumably irrelevant to the superconductivity).

The more general $N$-layer problem has been studied by Bulaevski and Zyskin 12 as well as Klemm and Liu.10 In these calculations, it was also assumed that the $c$-axis consisted of a coherently coupled stack of bi-layer structural units. Here we presume that the considerable evidence for the absence of $c$-axis coherence 2425 requires a different starting point. While, for simplicity, we focus on an isolated $N$-layer complex, it is clear that higher order effects associated with incoherent coupling between unit cells must ultimately be included. Such incoherent coupling can be introduced following, for example, Ref. 26. An additional complexity was raised in even earlier studies by Efetov and Larkin 27 who pointed out that a complete treatment of interlayer electron pairing required the consideration of a triplet pairing state odd under exchange of layer indices. This case was further investigated by Kettemann and Efetov 13 as well as Klemm and Liu 10 who argued that the mixing of triplet and singlet states would generally lead to a second transition below $T_{c}$. Thus far there is no evidence for this second transition, nor is there much support for the triplet pairing state.

There are important issues of controversy implicit in the work discussed here. There appears to be little doubt that the components of the bi-layer couple magnetically. However, it has not been persuasively demonstrated that there is a coherent electronic coupling $t_{\perp}$ within the unit cell.28 Neutron experiments 29 on 31 BCO provide evidence for a $c$-axis modulation associated with the spacing of the planes within the bi-layer. While this can be explained by magnetic coupling alone, 32,33 earlier photoemission studies on this cuprate have reported the observation of two copper oxide plane bands. 6 The situation in BSCCO is even less certain with two photoemission groups reaching opposite conclusions. purposes of the present paper it will be assumed that there is coherent coupling between the layers, though not between the unit cells. This assumption is based in large part on the demonstrated intra-bi-layer magnetic interactions which suggest a moderate degree of communication between the layers. Moreover, on this basis it may be presumed that there are direct or indirect electronic interactions within the bi-layer complex which must be included in any complete theory of the superconductivity.

Similarly controversial is the origin of a possible $d_{x^{2}-x^{2}}$ state. While this state is consistent with spin fluctuation mediated superconductivity, 134 in this paper we demonstrate that it appears more generally as a solution to the gap equation in the presence of repulsive 
interactions. Providing this repulsion has some wave-vector dependence (near, although not necessarily pinned at the anti-ferromagnetic position $(\pi, \pi)$ ), we find the $d_{x^{2}-y^{2}}$ state to be stable. Thus, experimental observation of this symmetry appears to provide more support for superconductivity arising from repulsive interactions than for any detailed superconducting mechanism. While there are problems associated with interpreting various experiments within a $d_{x^{2}-y^{2}}$ context, among the most perplexing from a theoretical viewpoint ${ }^{35}$. 36 is the evident impurity insensitivity of the superconducting transition temperature. 7 Moreover, substitution at the rare earth site in YBCO with both magnetic and non-magnetic atoms, between the planes of the bi-layer, leads to no variation in $T_{c}$, except in the special case of Pr. Here we address this fastinating puzzle and demonstrate that for the case of two out of phase nodeless $s$-states in an isolated bi-layer configuration, Anderson's theorem applies to inter-plane substitutions. This provides additional motivation for studies of this particular pairing state.

An outline of the paper is as follows. In Section II we focus on the bilayer problem as a prototype of the $N$-layer system. We introduce a generalized model for the pairing interaction (Section IIA) and then point out some general properties of solutions which arise in these systems (Section IIB). In Section $\mathbb{I I O}$ we examine the consequences of the bilayer structure on Josephson tunneling experiments between cuprates and conventional superconductors as well as the problem of twinned samples. We then construct (Section [ID) the free energy functional in the Landau-Ginzburg limit, addressing the effects of interlayer tunneling and orthorhombicity. Impurity scattering will be discussed in Section $\mathbb{1 T E}$, where we investigate the circumstances under which Anderson's theorem holds for the case of two out of phase $s$-states.

In Section [II] we turn to the more technical issues of a general weak coupling treatment of the problem of $N$ two dimensional layers. The reader uninterested in the more mathematical details can skip directly to the conclusions in Section $\mathbb{I V}$. The generalized gap equation in the $N$ layer problem is derived (Section $\Pi 1 \mathrm{~A}$ ) and the two competing states are discussed in Section IIIB. These correspond to natural analogues of the in-phase and out-of-phase states of the bi-layer system. We conclude with a general discussion (Section 【II) of the role of van Hove effects. Two states are found to take maximal advantage of the van Hove singularities: these are the $d_{x^{2}-y^{2}}$ and nodeless $s$-states. Some of the technical aspects of these calculations as well as a complete solution of the bilayer problem are relegated to Appendices A and B respectively.

Our conclusions are presented in Section IV.

\section{THE $N=2$ CASE}

\section{A. Gap Equation and Model Interaction}

We initially study the simpler bi-layer system $(N=2)$ in order to establish notation and to discuss physical results in a more familiar context. Although only singlet intraband pairing is considered in the main part of the text, a more complete weak coupling calculation of the bilayer problem will be presented in Appendix B. Many of the interesting features of bilayers arise in the general $N$-layer problem with only slight modification. However, this more general case will be discussed in detail in Section 【II. Section 【IB focuses primarily 
on the linearized gap equation. Throughout Section [1], particular attention will be devoted to elucidating the role of orthorhombicity.

The gap equations for two copper oxides planes are given by

$$
\begin{aligned}
& \Delta_{+}+\Delta_{-}=-\sum_{\mathbf{q}} V_{\|}\left(\Delta_{+} \frac{\tanh \frac{1}{2} \beta E_{+}}{2 E_{+}}+\Delta_{-} \frac{\tanh \frac{1}{2} \beta E_{-}}{2 E_{-}}\right) \\
& \Delta_{+}-\Delta_{-}=-\sum_{\mathbf{q}} V_{\perp}\left(\Delta_{+} \frac{\tanh \frac{1}{2} \beta E_{+}}{2 E_{+}}-\Delta_{-} \frac{\tanh \frac{1}{2} \beta E_{-}}{2 E_{-}}\right)
\end{aligned}
$$

where we define

$$
E_{ \pm}^{2}=\epsilon_{ \pm}^{2}+\left|\Delta_{ \pm}\right|^{2}
$$

This equation was previously discussed in Ref. 8. Its general derivation is presented in the $N$-layer context in Section ШIIA. The two order parameter components $\Delta_{+}$and $\Delta_{-}$ refer to pairing on the bonding and anti-bonding bands of the Fermi surface respectively. Throughout this paper we will transform between the band and layer indices. The two gap parameters may be related to order parameters in the layer language by the equations

$$
\begin{aligned}
& \Delta_{+}=\Delta_{\|}+\Delta_{\perp} \\
& \Delta_{-}=\Delta_{\|}-\Delta_{\perp}
\end{aligned}
$$

where $\Delta_{\|}$refers to singlet pairing of electrons within individual layers and $\Delta_{\perp}$ refers to singlet pairing of electrons on different layers. Finally, the normal state energy dispersion is given by $\epsilon_{ \pm}=\xi \mp t_{\perp}$, where $t_{\perp}$ is the interlayer hopping matrix element and $\xi(\mathbf{q})$ is the single particle dispersion within the copper oxide planes. It should be noted that allowing the interlayer tunneling to depend on $\mathbf{q}$ in the manner suggested by Chakravarty and Andersonet as well as Andersen et al. .38

$$
\frac{t_{\perp}}{4}\left(\cos q_{x}-\cos q_{y}\right)^{2}
$$

has no qualitative effect on the results of this paper. This arises because for the model of Ref. 28 the inter-plane tunneling deviates most from the constant value $t_{\perp}$ along the diagonals of the Brillouin zone (where Eq. (4) vanishes). It is in precisely this region where the density of states contributions are smallest, since, as will be discussed in more detail below, they lie away from the four van Hove points.

As noted above, the bilayer system admits two other pairing states, an interlayer triplet state $\Delta_{\perp}^{t}$ which is odd under interchange of layer labels and a second intralayer singlet state $\Delta_{\|}^{o}$ where the order parameter has opposite phase on each of the two layers. Both of these correspond to inter-band pairing, i.e. pairing between electrons on different sub-bands of the Fermi surface. Thus they appear as off-diagonal elements of $\Delta$ in the band representation. Because this inter-band pairing is associated with a second phase transition (when $t_{\perp}$ is finite) as well as a triplet state, it appears to be currently of less physical interest than the singlet, intra-band pairing which we consider here. For the purposes of completeness, these more exotic states are considered in Appendix B.

While our analysis of Eq. (1) is expected to be generally valid, we present here a weak coupling treatment, in large part because our emphasis is on the symmetry of the order 
parameter, rather than on the detailed values of the transition temperatures. Furthermore we adopt a model interaction which is believed to be sufficiently generic so as to encompass most pairing mechanisms in the literature. Thus it includes the well studied spin fluctuation interaction which is strongly peaked at quasi-momentum transfers of $\mathbf{Q}=(\pi, \pi), \mathbb{1}$ as well as phonon based mechanisms. 39 Alternative scenario 40 may be associated with intermediate $\mathrm{Q}$ values. In the present model, the in plane and inter-plane pairing interactions are given by

$$
\begin{aligned}
V_{\|}\left(\mathbf{q}, \mathbf{q}^{\prime}\right) & =\lambda_{\|} \chi_{\mathbf{Q}_{0}}\left(\mathbf{q}-\mathbf{q}^{\prime}\right) \\
V_{\perp}\left(\mathbf{q}, \mathbf{q}^{\prime}\right) & =\lambda_{\perp} \chi_{\mathbf{Q}_{0}}\left(\mathbf{q}-\mathbf{q}^{\prime}\right)
\end{aligned}
$$

where we define the generalized susceptibility, which depends on the momentum transfer

$$
\chi_{\mathbf{Q}_{0}}(\mathbf{q})=\frac{1}{h} \sum_{\mathbf{Q}=R \mathbf{Q}_{0}} \frac{1}{\left[1-J_{0}\left(\cos \left(q_{x}-Q_{x}\right)+\cos \left(q_{y}-Q_{y}\right)\right]^{2}\right.}
$$

This model incorporates several parametrizations, allowing the variation of the peak location through $\mathbf{Q}_{0}$, the peak strength via $J_{0}$, and the coupling strength and sign through $\lambda$. We consider $J_{0}$ to be below the critical value 0.5 . The interaction contains the underlying symmetry of the lattice. This is imposed by summing over all elements $R$ of the point group, $h$ being the order of the group. Thus when $\mathbf{Q}_{0}$ does not lie on a symmetry element of the lattice then the interaction will have eight peaks in a tetragonal $D_{4 h}$ lattice and four peaks in an orthorhombic lattice.

Our aim is to incorporate some of the key features of the known band structure in various cuprates. Thus the lattice point group will be assumed either to be $D_{4 h}$ in the case of LSCO or $D_{2 h}$ in the case of YBCO and BSCCO. We investigate two different kinds of broken tetragonal symmetry in which either principle axes are retained as symmetry planes (orthorhombic) in the case of YBCO or the diagonals are retained as planes of symmetry (rhombohedral) 1 in the case of BSCCO. 22 The band structures which we use throughout this paper are taken from Ref. 13. In plane energy dispersions of the normal state are given by an expansion of the form

$$
\xi(\mathbf{q})=\sum_{i=0}^{7} t_{i} \eta_{i}(\mathbf{q})
$$

where the basis functions $\eta_{i}$ are listed in Table $₫$. Also indicated in the table are the various band structures we will be considering. It is important to note that the degree to which the tetragonal $\left(D_{4 h}\right)$ symmetry is broken is reflected in the last two parameters of the table which we call $t_{6}$ and $t_{7}$. The size of these orthorhombic contributions was chosen, for illustrative purposes, to be small, but otherwise arbitrary.

\section{B. Order Parameter Symmetry in Bilayers}

\section{Properties of Mono-layer Solutions}

Solutions to the single layer problem provide intuition into those of the $N$-layer case, particularly when the interlayer hopping is relatively small. It is useful, therefore, to characterize the allowed order parameters in the one layer limit for each of the three bandstructures 
discussed above, before moving to the bi-layer case. In the one layer system the functional form of the superconducting order parameter depends only on the sign and the shape (as distinct from the magnitude) of the pair interaction and on the electronic structure. To illustrate these pair interaction and electronic effects, we vary the position of the peak location $\mathbf{Q}$ and plot the associated form of the order parameter in this two dimensional space, for each of three model bandstructures. The interaction is assumed repulsive $(\lambda>0)$ in this and subsequent phase plots; attractive interactions in general give rise to nodeless $s$-wave solutions for any value of $\mathbf{Q}$. The magnitude of the interaction plays no role in determining the symmetry of the order parameter in the single layer problem, it results only in a variation of the transition temperature. By contrast, in the two layer case the (relative) magnitudes of the in plane and out of plane interactions enter via Eq. (1) in an important way. We will examine this effect subsequently.

Other parameters of our model interaction have a lesser influence on the form and regions of stability of the various solutions. Variation of the peak width $J_{0}$ affects $T_{c}$ to a greater extent than it does the symmetry of the actual solution. The component of the interaction which most influences the symmetry of the superconducting order parameter is the degree to which the vector $\mathbf{Q}$ connects positive and negative lobes of the pair wave function on the Fermi surface. 34 As will be illustrated under more general circumstances below, near half filling, when only nearest neighbour hopping is included, the points at which the $d_{x^{2}-y^{2}}$ state has maxima are separated by $\mathbf{Q}=(\pi, \pi)$, while the extrema of $d_{x y}$ are separated by $(\pi, 0)$ and the eight lobed $s_{x y}$ state has positive and negative lobes separated by $(\pi / 2, \pi / 2)$. These are precisely the points in the space of $\mathbf{Q}$ near which these various solutions are favoured.

Figure 1 represents this "phase diagram" for the LSCO case. It is a map of the irreducible representations of the tetragonal, $D_{4 h}$ lattice which correspond to the solutions with the highest $T_{c}$. We stress that the simple functional forms of the order parameter, such as are implicit in the notation " $d_{x^{2}-y^{2}}$ " etc., can be misleading. The gap parameters may involve important contributions from higher order terms in the associated representation. Moreover when tetragonal symmetry is broken, states may be highly admixed with states of other representations. We will use this notation with care, applying it only to certain solutions of systems with tetragonal symmetry. When necessary we will avoid any ambiguity by using the common group theoretic nomenclature for the various irreducible representations. 4

Because proximity to the van Hove singularity is found to play such a crucial role, we present two limiting cases corresponding to choices for the Fermi energy near ([1]a) and far from (1]b) the van Hove singularity. The order parameters shown plot the actual solutions for $\mathbf{Q}=(\pi, \pi)$, at which the $B_{1 g}\left(" d_{x^{2}-y^{2}}\right.$ ") solution is found, at $\mathbf{Q}=(\pi / 2, \pi / 2)$, where an eight-lobed $A_{1 g}$ solution, the so called "s $s_{x y}$ " state, exists and at $\mathbf{Q}=(\pi, 0)$, where the $B_{2 g}$ " $d_{x y}$ " state is most stable. Moreover, these symmetries persist over an extended region of Q-space as outlined by the solid lines in the figures. States with increasing numbers of nodal planes, including states in the $A_{2 g}$ irreducible representation, appear as $\mathbf{Q} \rightarrow(0,0)$. Indeed, because this region is so complicated (in the case of repulsive interactions), no detailed solutions are indicated. The region where the " $d_{x^{2}-y^{2}}$ "-like solution persists is shaded in this and subsequent figures.

Figures (17a) and (11b) demonstrate that proximity to the van Hove singularity leads to a modestly extended region with $B_{1 g}$ or " $d_{x^{2}-y^{2}}$ " symmetry. Moreover as the Fermi energy approaches the singularity, the lobes on the " $d_{x^{2}-y^{2}}$ " state become sharper. In this way the 
order parameter takes full advantage of the four van Hove points which coincide with each of the four maxima in this gap function. Since the gap function is sharply peaked in the direction of the principle axes it is very small in a large region about the nodal lines along the diagonals of the Brillouin zone. A large gapless region will have consequences on the thermodynamic properties of the samples at low temperatures and may have been observed in ARPES measurements on BSCCO呞

Breaking of the tetragonal symmetry, as in the case of the orthorhombic lattice of YBCO, serves to further enhance these van Hove effects. This is shown in Figure 2 where, as in the LSCO case above, the gap equation solutions are plotted at the special symmetry points and the lines indicate the boundaries of the region over which the representative solution persists. Solid lines separate states belonging to different irreducible representations and dotted lines separate states of different symmetry within a representation. Figures (2a) and (2/b) demonstrate clearly that the phase space occupied by the " $d_{x^{2}-y^{2}}$ " solution is dramatically increased in the vicinity of the van Hove point. Furthermore, the lobes of this gap function again become more extended along the principle axes, compared with similar solutions farther from the van Hove point.

Finally, the results in the BSCCO case are presented in Figure 3. We see here that, contrary to the two previous cases, the phase space in which the gap has the same sign along the $a$ and $b$ directions (i.e, the totally symmetric irreducible representation in the $D_{2 h}$ lattice) occupies a somewhat larger region of parameter space when the Fermi level is near the van Hove point. This is the consequence of a stabilizing admixture of an isotropic $s$-wave component. On the other hand, states with an $a, b$-axis $\pi$ phase shift dominate the phase space away from the van Hove points.

The above three figures lead to an important conclusion: the " $d_{x^{2}-y^{2}}$ " solution exists in a large region of parameter space away from $\mathbf{Q}_{\mathrm{AF}}$. Therefore this state should be considered as an obvious candidate form for the order parameter in any mechanism involving pairing via a predominantly repulsive interaction. Stated alternatively, observation of this form for the gap in an experiment is in itself not proof of any specific pairing mechanism. Furthermore, it also appears from these figures that, quite frequently, a single mechanism can give rise to widely varying forms of order parameters depending upon the details of the band structure, doping level, lattice symmetry and other subtle features of the material.

\section{Bi-layer Effects}

In bi-layer materials there are two possible states which compete as solutions to the gap equation. These may be crudely classified by referring to the states as "inter-plane and intra-plane dominated solutions". These two competing sets of solutions were discussed in a

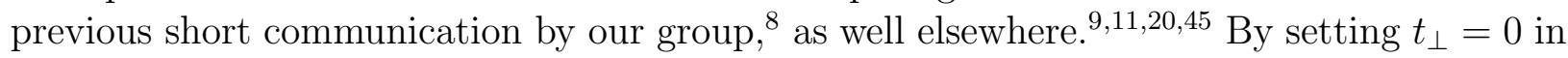
the gap Eq. (11) we see that $\Delta_{\|}$and $\Delta_{\perp}$ uncouple from each other at $T_{c}$. Superconductivity can thus be either due to interlayer pairing or due to intralayer pairing depending on the relative magnitudes and signs of $\lambda_{\|}$and $\lambda_{\perp}$. In the band representation in-plane pairing yields a solution which has the same phase on both bands while the interlayer pairing solution undergoes a $\pi$ change in phase from one band to the other. This sign change follows directly from Eq. (3) in the extreme limits in which one or the other order parameter (in the layer basis) is the larger. 
It is important to stress that we will refer to these two competing solutions throughout this paper. They occur in bi-layer as well as $N$-layer systems. In all cases where $t_{\perp}$ is finite, one solution is stable and the other metastable. Thus, although one may refer to their associated transition temperatures, only the larger of the two has any physical meaning. As a consequence, there is nothing to prevent these two competing states from having a different order parameter symmetry.

As in the one layer case, repulsive interactions are found to lead to nodal states such as the $d$-states or multi-lobed $s$-states discussed above, while attractive interactions require nodeless states of $s$ symmetry. In the presence of two different types (signs) of in plane and out of plane interactions, these effects provide a rich and complex structure for the competing order parameter states. Under these conditions and when there is no coherent inter-plane hopping, the two solutions may belong to different symmetry groups. When $t_{\perp}$ is finite, the solutions couple while still preserving the underlying lattice symmetry. Thus mixing of $s$ - and $d$-states within the linearized gap requires both orthorhombicity and interplane hopping. More general $s$ - $d$ mixing effects will occur at higher (quartic) order in a Landau-Ginzburg expansion, and will be discussed in more detail in a subsequent section.

To illustrate the behavior of the bi-layer order parameter and the two competitive states, we consider for concreteness the case of an attractive interplane interaction and a repulsive intraplane interaction peaked at $\mathbf{Q}=(\pi, \pi)$. This model may be viewed as derived from a generalized spin fluctuation model, where the inter-layer or $c$-axis spin susceptibility is included as a perpendicular component of the pairing interaction. This case was previously discussed by a number of different groups.6.11 45 . 4 . While we choose this peak location simply for illustrative reasons, our results can easily be extended to other values of $\mathbf{Q}$. Under these circumstances, the order parameter in the band picture has the schematic form

$$
\left(\begin{array}{c}
\Delta_{+} \\
\Delta_{-}
\end{array}\right)=\left(\begin{array}{c}
s_{\perp}+d_{\|} \\
-s_{\perp}+d_{\|}
\end{array}\right)
$$

If the lattice is tetragonal then either $s_{\perp}$ or $d_{\|}$will vanish. Thus when $\lambda_{\perp}$ is zero the solution will have $d$-symmetry. Moreover, both band gaps are in phase. Similarly when $\lambda_{\|}$vanishes the symmetry is $s$-like and both gaps are out of phase. The transition from one type of solution to the other is governed by size of $\lambda_{\perp} / \lambda_{\|}$. Frequently the cross over from the inplane to the inter-plane dominated regime may occur despite the fact that the ratio $\lambda_{\perp} / \lambda_{\|}$ is small compared to one. What determines this cross-over is the nature and sign of the competing interactions and the bandstructure.

If tetragonal symmetry is broken even more complicated situations obtain. Both components can be nonzero simultaneously and the transition is a smooth one as a function of increasing $\lambda_{\perp} / \lambda_{\|}$. The regions where the various types of solution exist in YBCO are illustrated in Figure $\$$ for the example discussed above. Plotted on the vertical axis is the fractional hole doping $x$. We estimate the physical values of this parameter, for a range of different oxygen stoichiometries in YBCO to vary from 0.1 to 0.3. The figure illustrates the parameter regimes where the dominant component of the order parameter on the two bands is $(d, d),(s, d)$ and $(s,-s)$.47 The region below the solid line involves states in which the sum of the pair wave functions on the two bands changes sign under $\pi / 2$ rotation of the axes and can thus yield $\pi$ phase shifts in $a, b$-axis Josephson tunneling experiments $14-17$ Moreover, the shaded regions denote states in which the sum of the two order parameters changes sign 
even though one or both are nodeless. This issue will be addressed in detail in Section 『IC. The inset plots the density of states as a function of energy. There are actually four van Hove points associated with orthorhombic splitting of the two bands. For clarity the doping level at which the first of these occurs is plotted in the main portion of the figure as a dotted line. Note that the region labelled by $(s,-s)$ occupies the largest fraction of phase space when the band is near the van Hove points in general and in particular when one sub-band of the Fermi level is closed about the $\Gamma$ point of the reciprocal lattice and the other about $X$.

The evolution of these solutions with increasing $\lambda_{\perp} / \lambda_{\|}$is illustrated in Figure 5 . Indicated here is the calculated shape of the gap functions for the two bands of the bi-layer. Their relative phase is also noted. It can be seen that the pair wave function on each band has a greater amplitude along one symmetry axis than along the other, and the axis along which the pair wave function has its maximum differs from one sub-band to the other. These results are plotted for very small orthorhombicity and for Fermi energies close to the van Hove point. On the basis of the latter assumption, the small orthorhombic effects are greatly magnified. The figure labels (a)-(d) correspond to (a) two in phase $d$-wave dominated states, (b) one band nodeless and the other nodal, (c) two out of phase nodeless states which show greatly elongated lobes, and (d) to two out of phase $s$-wave dominated states which are more isotropic. The relative phase space which these solutions occupy can be seen from the previous figure.

Moreover, the detailed shape of the order parameter has important physical consequences. Thus for case (c), for example, the solutions are in the inter-plane regime where $\Delta_{\perp}$ is dominant. Here, however, the order parameter is nodeless and yet the combination of the two gaps has features of a $d$-wave solution in $a, b$-axis as well as $c$-axis Josephson tunneling experiments. $\$$ Case (b) is associated with one $s$-like and one $d$-like state. It will exhibit both power law behavior in thermodynamical properties, as well as the $\pi$ phase shifts in corner junction experiments, which are also seen in cases (a) and (c). These Josephson experiments and their relation to the order parameter symmetry will be discussed in more detail in the next section.

\section{Josephson Tunneling: Phase Coherence Across Domain Boundaries}

Josephson tunneling experiments have been key to elucidating the order parameter symmetry in the YBCO cuprate family 14.7 .6 Within this class, two types of measurements have been performed. These involve $a, b$ - and $c$-axis tunnel junctions. In the former category SQUID geometries have been investigated.15 17 These consist of two junctions with $\mathrm{Pb}$ counterelectrodes, whose interference pattern yields information about the relative phase of the order parameter along various axes in the $a, b$-plane. In "corner junctions", $\pi$ phase shifts of the order parameter between the $a$ and $b$ axes have been observed and attributed to the sign change upon a $\pi / 2$ rotation of the wave vector, within a $d_{x^{2}-y^{2}}$ state. This interpretation is further confirmed by "edge" junctions which probe the interference along a single face of the material; here no phase shifts are found to be present. These conclusions are the same for both twinned and untwinned crystals. A variant on this geometry are the ring experiments 1 tensisting of YBCO segments with different grain boundary orientations. Observation of a non-zero, half integer spontaneous flux threading the ring, for 
specific orientations of the grain boundaries, again provides support for a $d_{x^{2}-y^{2}}$ state.

An alternate Josephson tunneling geometry has been investigated by Sun et al 48 These authors observe finite $c$-axis Josephson currents between a $\mathrm{Pb}$ counterelectrode and a YBCO sample. For the simplest representation of the $d_{x^{2}-y^{2}}$ symmetry, no Josephson current should be present. While it is possible to invoke orthorhombicity 49 to explain these non- vanishing results, this hypothesis seems unlikely since the magnitude of the current is evidently not sensitive to whether twins are present.

To the extent that twinned YBCO can be treated as a tetragonal system, these two types of experiments appear manifestly incompatible. $\pi$ phase shifts are associated with a sign change of the order parameter in the $a, b$ - plane. The $c$-axis current, which reflects an average of the order parameter in the $a, b$-plane, must necessarily vanish. These observations are not altered when bi-layer effects are incorporated. While there has been some attention 2123 paid to the role of chains in addressing the data, it should be noted that recent experiments on reduced oxygen YBCOL5 (where the chains are expected to be highly interrupted and, therefore, irrelevant to the transport and superconductivity), seem to reproduce the same behavior as in the optimally doped material. In summary, if both $a, b$ - and $c$-axis Josephson measurements prove to be correct, any resolution of this issue will probably revolve around a deeper understanding of twinning effects.

In a Josephson experiment, the measured Josephson current is the sum of the currents established between each band of the cuprate and the superconductor to which it is coupled

$$
J=J^{+}+J^{-}
$$

where each component has the usual Ambegaokar-Baratoff form巨

$$
J^{ \pm}=\Delta_{ \pm}^{L} \Delta^{R} \frac{\pi}{\beta r_{ \pm}} \sum_{n}\left[\left(\omega_{n}^{2}+\Delta_{ \pm}^{L^{2}}\right)\left(\omega_{n}^{2}+\Delta^{R^{2}}\right)\right]^{-\frac{1}{2}}
$$

The resistances, $r_{ \pm}$, can be calculated by determining the single particle tunneling matrix elements 11

$$
T_{k, k^{\prime}}^{ \pm}=\int \operatorname{dr}\left\langle\phi_{k, \pm}^{L}|j(r)| \phi_{k^{\prime}}^{R}\right\rangle
$$

where the integration is carried out over the intermediate region. The wave-functions $\phi_{ \pm}^{L}$ and $\phi^{R}$ are the single particle wave-functions from the left and right hand side of the junction respectively, and $j(r)$ is the usual current operator. The fact that the electrons of the cuprates are localized on the copper-oxide planes requires that they be described by wave packets with some finite spread in momentum along the $c$ - axis direction. A microscopic treatment of the tunneling process is complicated by a number of issues, which we cannot address here: the propagation of electrons out of a region of localization in the $c$-direction will lead to scattering effects as the tunneling pair enters a more isotropic material. These may influence the tunneling from both bands to a substantial degree. (It should also be noted that the order parameter at the surface may be modified from its bulk form.52) For definiteness, we first consider the case of coupling to a conventional superconductor such as $\mathrm{Pb}$, and ignore these complications.

If we make the simplifying assumption that the Josephson coupling is the same for both the symmetric (+) and antisymmetric (-) bands, it follows that $\pi$ phase shifts in a 
SQUID experiment will be observed in a substantial region of the phase space, corresponding to all bi-layer parameter sets which lie below the solid line of Figure 1 . Moreover, for a significant fraction of these (particularly in the vicinity of the van Hove points), the states in question differ from two in phase $d$-like states. They are described by $(s, d)$ or $(s,-s)$ dominant combinations. In this way, the measurement of $\pi$ phase shifts cannot be uniquely associated with $d$ states in a multi-layer system, provided orthorhombicity is also present. This observation makes it all the more important to repeat the various SQUID experiments for either mono-layer or tetragonal materials.

In reality, there is some asymmetry in the Josephson coupling to the symmetric and anti-symmetric bands. This asymmetry derives from (1) density of states effects related to the van Hove points and (2) wave- function structure: wave functions associated with the anti-bonding band of the bilayer cuprate have opposite phase on the two layers and therefore a nodal plane exists between the layers. The first effect tends to enhance Josephson coupling preferentially to the antibonding and the second to the bonding band. The net contribution cannot be calculated with any certainty, but it is reasonable to conclude that the Josephson coupling is appreciable for both bands of the bi-layer system.

These remarks can be addressed in somewhat more detail. In most of the high $T_{c}$ cuprates for which band structure data is available, the Fermi surfaces are closed about the $(\pi, \pi)$ point. As a consequence the anti-bonding band lies closer to the van Hove points and thus has a higher density of states. This density of states contribution will then lead to a relatively stronger Josephson coupling for the anti-bonding or antisymmetric band. On the other hand, the amplitude of the pair wave function is expected to be greater on the even symmetry band 53 and this effect may lead to a greater contribution to the Josephson current than for the even band. Moreover, it has been claimed 54 on the basis of symmetry arguments, that tunneling from the anti-bonding band into an $s$-wave superconductor will lead to an appreciable reduction in the current contribution from this band. However, some Josephson coupling is expected to remain; the matrix elements $T_{k, k^{\prime}}^{-}$only vanish under the special circumstances, when the centers of the two wavefunctions of Eq. (11) co-incide throughout the boundary region. In general, this matrix element will be nonzero, if for no other reason than because the lattice constants of the two materials are unlikely to be commensurate. The situation is depicted in Figure 6 .

Josephson tunneling from one YBCO crystal to another, as well as across twin boundaries, is even less amenable to microscopic theory. In many respects twinned materials behave like single crystals, with similar transition temperatures, thermodynamics and Josephson currents in corner and $c$-axis junctions. It is clear that little is understood at a detailed theoretical level about the nature of the twin boundary. What seems to be less ambiguous, however, is that there is little if any pinning of the critical current (in low magnetic fields) as it flows between twin boundaries.5 This would suggest that, whatever the order parameter symmetry, the phases tend to line up with + and - lobes adjacent.

In the context of a bi-layer system, this situation is more complex, since the phases are associated with multiple bands. In Figure 7, we schematically plot the two alternative scenarios for the $(s,-s)$ (orthorhombic) bands (parts (b) and (c)), as well as the generally expected behavior for the $(d, d)$ case (a). Of the two scenarios (b) and (c), only the latter would preserve the $\pi$ phase shift behavior across a twin boundary. This scenario would also be compatible with a low twin boundary pinning of the critical current. 
Despite these phenomenological arguments, case (b) cannot be ruled out on microscopic grounds. In the extreme limit where the boundary can be treated as a conventional SIS junction, the locking of the phase of the order parameter across the junction occurs via Josephson coupling. If the bilayers on either side of the junction are properly aligned it might be expected that only bands of equal symmetry couple together since the matrix element in Eq. (11) vanishes otherwise. Thus the even bands on either side of the junction would couple together as would the odd bands. As a consequence the positive lobes of a $\left(\Delta_{+}, \Delta_{-}\right)=(d, d)$ solution would point in the same spatial direction on both sides of the twin boundary(scenario (a)). On the other hand the $(s,-s)$ state would have the component of positive phase in one twin aligned with the component with negative phase in the other twin (scenario (b)). This would lead to a substantially reduced Josephson current at a macroscopic junction due to averaging over the twins. If instead of an SIS model, one argued that the order parameter lobes were required to vary in the most continuous fashion, one might conclude that the dominant component $\Delta_{\perp}$ should also be continuous. This, too, would lead to scenario (b) for the $(s,-s)$ states and (a) for the $(d, d)$ configuration.

Nevertheless, it is also possible to assume that interlayer pair breaking effects become considerable in the boundary region and that $\Delta_{\|}$retains its coherence between twins so that scenario (c) obtains. Moreover, stacking faults, lattice defects and other complexities can invalidate any of the above simple models of twinned crystals. In summary, the nature of the order parameter variation across a twin boundary is quite complicated in one layer systems and sufficiently complex in the bi-layer case so that no clear conclusions can be drawn at this time.

\section{Landau-Ginzburg Free Energy Functional}

Thus far we have investigated only the linearized gap equations, which are necessarily restricted to the vicinity of the transition temperature. Additional effects may occur below $T_{c}$ associated with the transition to states with other order parameter symmetries. While there does not appear to be experimental evidence for additional phase transitions, there is considerable information contained in studying the more general situation. In this section we derive the appropriate Landau-Ginzburg free energy functional in terms of both the layer and band indices. The behavior at quadratic order gives further insight into, and serves to validate the results discussed in the previous sections. There have been several discussions in the literature 20 of phenomenological forms for the bi-layer free energy. Here we proceed from a microscopic basis. Note that while this discussion refers to a bilayer structure, it is readily extended to the case of general $N$ following the results of Section IIIB.

In the case under consideration (when only pairing of electrons on individual sub-bands of the Fermi surface is considered), the superconducting state of the bi-layer is described by a two component order parameter : $\Delta_{+}$and $\Delta_{-}$. The free energy, $F_{s}$, of the superconducting state then consists of the sum of terms

$$
F_{s}=F_{s,+}+F_{s,-}
$$

where $F_{s, \pm}$ is the contribution to the free energy of an individual sub-band. In the LandauGinzburg limit the free energy difference between the normal and superconducting states is given by 


$$
F_{s, \pm}-F_{n, \pm}=\ln \frac{T}{T_{c}} \int_{\epsilon_{ \pm}=E_{F}} \frac{\mathrm{d} S}{(2 \pi)^{2} v_{F}}\left|\Delta_{ \pm}\right|^{2}+\frac{0.0533}{\left(k_{B} T_{c}\right)^{2}} \int_{\epsilon_{ \pm}=E_{F}} \frac{\mathrm{d} S}{(2 \pi)^{2} v_{F}}\left|\Delta_{ \pm}\right|^{4}
$$

It should be stressed that, although the two sub-bands contribute independently to the free energy, the two order parameters are coupled via the gap equation. Both become finite at a common $T_{c}$.

By use of the gap equations the the Landau-Ginzburg free energy functional can be recast into a form which contains a quadratic part

$$
\alpha_{+}\left|\Delta_{+}\right|^{2}+\alpha_{-}\left|\Delta_{-}\right|^{2}+\delta\left(\Delta_{+} \Delta_{-}^{*}+\text { c.c. }\right)
$$

and a quartic contribution

$$
\beta_{+}\left|\Delta_{+}\right|^{4}+\beta_{-}\left|\Delta_{-}\right|^{4}+\left(\Delta_{+} \Delta_{-}^{*}+\text { c.c. }\right)\left(\mu_{+}\left|\Delta_{+}\right|^{2}+\mu_{-}\left|\Delta_{-}\right|^{2}\right)
$$

Here we have assumed that each component of the order parameter can be separated into a complex magnitude times a normalized real function over the Fermi surface:

$$
\Delta_{ \pm}(\mathbf{q})=\Delta_{ \pm} \psi_{ \pm}(\mathbf{q})
$$

The magnitude $\Delta_{ \pm}$appears explicitly in the Landau-Ginzburg free energy expansion while $\psi_{ \pm}$determines the co-efficients in the expansion. The co-efficients $\delta$ and $\mu_{ \pm}$vanish if $\Delta_{+}$ and $\Delta_{-}$belong to different irreducible representations of the lattice point group.

These coefficients have been discussed by Varma in the context of a particular pairing scenario. .0 While our free energy contains the same class of terms as that presented in Ref. 40 , because we have explicitly removed the wave vector dependences via Eq. (16) above, it is not straightforward to determine the conditions for order parameter sign changes under a $\pi / 2$ rotation of the lattice. Nevertheless, the relative phase of the two order parameters can be determined variationally, by minimizing the free energy. If the nontrivial coupling parameters $\left(\delta\right.$ and $\left.\mu_{ \pm}\right)$are finite then the phase difference between $\Delta_{+}$and $\Delta_{-}$can be at most 0 or $\pi$. This last result is consistent with the discussion of Section $11 \mathrm{B2}$ and the appropriate sign depends on whether in-plane or interplane correlations are dominant 56

It is useful to transform the above free energy functional to the layer representation using Eq. (3). The functional is expressed in terms of $\Delta_{\|}$and $\Delta_{\perp}$ and two explicit transition temperatures can then be associated with the intra- and inter-plane interactions. In this context the mixing between various symmetries can be understood in a more direct way. In this representation the free energy is given by

$$
\begin{gathered}
\alpha_{\|}\left|\Delta_{\|}\right|^{2}+\alpha_{\perp}\left|\Delta_{\perp}\right|^{2}+\delta^{\prime}\left(\Delta_{\|} \Delta_{\perp}^{*}+\text { c.c. }\right)+\beta_{\|}\left|\Delta_{\|}\right|^{4}+\beta_{\perp}\left|\Delta_{\perp}\right|^{4}+\beta^{\prime}\left|\Delta_{\|}\right|^{2}\left|\Delta_{\perp}\right|^{2} \\
+\gamma^{\prime}\left(\Delta_{\|}^{2} \Delta_{\perp}^{* 2}+\text { c.c. }\right)+\left(\Delta_{\|} \Delta_{\perp}^{*}+\text { c.c. }\right)\left(\mu_{\|}\left|\Delta_{\|}\right|^{2}+\mu_{\perp}\left|\Delta_{\perp}\right|^{2}\right)
\end{gathered}
$$

Here the coupling terms $\delta^{\prime}$ and $\mu_{\| / \perp}$ vanish if $\Delta_{\|}$and $\Delta_{\perp}$ belong to different irreducible representations of the lattice symmetry group. Note that one important effect of orthorhombicity is to require that the coupling parameters $\delta^{\prime}$ and $\mu_{\| / \perp}$ change sign upon the interchange of the $a$ and $b$ axes of the crystal. These same terms also vanish in the limit as $t_{\perp}$ goes to zero.

By direct calculation from the microscopic theory we find that the quartic cross-term $\gamma^{\prime}$ is positive 5 so that the phase difference between $\Delta_{\|}$and $\Delta_{\perp}$ is zero (if $\delta^{\prime}<0$ ) or $\pi$ (if 
$\left.\delta^{\prime}>0\right)$. 5 Studies of a related Landau-Ginzburg free energy functional have been presented by Kobuki and Lee 20 who discussed the mixing between a $d$-wave $\Delta_{\|}$and an $s$-wave $\Delta_{\perp}$ in an RVB based theory. In their approach, mixing between these two components was brought about by a self-consistently determined orthorhombic strain. This led to the introduction of additional terms into the free energy which could result in a negative $\gamma^{\prime}$. As a consequence an $s+\iota d$ state was produced. In the present theory, in contrast, mixing between $\Delta_{\perp}$ and $\Delta_{\|}$ occurs at quadratic rather than quartic order, as a consequence of finite $t_{\perp}$. Furthermore, in the absence of tetragonal symmetry breaking only $s+d$ mixing occurs (at quartic order). Additional arguments against an $s+\iota d$ state were presented by Normand et al. $\omega^{6}$

\section{E. Impurity Effects}

In this section we discuss the nature of impurity or pair-breaking effects in bi- layer systems. At the heart of this issue is the paradoxical observation that all substitutions at the rare earth site, which sits between the bi-layers (except for Pr), leave $T_{c}$ unaffected. Rare earth substitutions with or without local moments and in a disordered or ordered form make no difference to the superconducting transition temperature. Previously it has been argued that the even more general insensitivity of the cuprates to impurity substitution is incompatible with anisotropic or $d$-wave superconductivity. 3 complexity introduced into this problem by the presence of a bi- layer order parameter. Since it is relatively straightforward to generalize to the magnetic case, for definiteness, we concentrate on the case of non- magnetic impurities.

A new aspect of the present work is the consideration of an isolated bi-layer, rather than a coherent stack of bi-layers. Our starting point is a necessary first step in a treatment of incoherent coupling along the $c$-axis. As a result of this assumption the configuration averaging process (which restores the underlying translational symmetry of the lattice) is different from that discussed in Refs. 13,59. As has been noted elsewhere, 13 there are two types of impurities which must be considered: intra and inter-layer substitutions. For an isolated bi-layer, inter-layer impurities represent the more interesting case, since the relevant equations decouple when written in the band basis. Thus a state such as an isotropic $(s,-s)$ state will be insensitive to inter-layer impurities (as will the in-phase $(s, s)$ state). By contrast, intra-layer impurity effects involve processes which couple the two bands. In this way, the $(s,-s)$ state exhibits intra-layer pair-breaking. 13 Moreover, the same concerns that were raised earlier 36 . 3 about the $d$-wave order parameter apply to the bi-layer case with either type of impurity. Thus the $(s,-s)$ state emerges as the leading (non-trivial) candidate state for resolving the paradox concerning rare earth (non-magnetic) substitutions in the cuprates.

We begin with the standard treatment of scatterers within individual planes using the Born approximation 60 The impurity Hamiltonian has the form

$$
\hat{H}_{\mathrm{inp} \text {-plane }}^{\mathrm{imp}}=\sum_{m} \sum_{\mathbf{q}, \sigma} u_{I}(\mathbf{q}) c_{i_{m}, \sigma}^{\dagger}(\mathbf{p}+\mathbf{q}) c_{i_{m}, \sigma}(\mathbf{p}) e^{-\iota \mathbf{q} \cdot \mathbf{R}_{m}}
$$

where $m$ labels impurities located at $\mathbf{R}_{m}$ on layer $i_{m}$ and $\sigma$ is a spin index. The impurity self-energy is given by 


$$
\Sigma_{\omega, r, r^{\prime}}(\mathbf{p})=-\frac{1}{2} n_{\text {imp }} \sum_{r^{\prime \prime}, \mathbf{q}}\left|u_{I}(\mathbf{p}-\mathbf{q})\right|^{2} \overline{\mathcal{G}}_{\omega, k^{\prime \prime}}(\mathbf{p}) \delta_{r, r^{\prime}}
$$

where $\overline{\mathcal{G}}$ is the averaged Green's function and $n_{\text {imp }}$ the concentration of impurities per layer. We assume that the gap in the absence of impurities is given by $\Delta_{ \pm}(\mathbf{q})=\bar{\Delta}_{ \pm} \psi_{ \pm}(\mathbf{q})$ where $\psi_{ \pm}$are functions normalized appropriately over the corresponding sub-band of the Fermi surface and that the impurity renormalized gap is given by $\tilde{\Delta}_{ \pm} \psi_{ \pm}(\mathbf{q})$. The self consistent equations then become

$$
\begin{gathered}
\tilde{\omega}_{l}=\omega_{l}+\frac{1}{4 \tau} \sum_{r^{\prime}= \pm} \frac{\tilde{\omega}_{l}}{\sqrt{\tilde{\omega}_{l}^{2}+\tilde{\Delta}_{r^{\prime}}^{2}}} \\
\tilde{\Delta}_{ \pm}=\bar{\Delta}_{ \pm}+\frac{g}{4 \tau} \sum_{r^{\prime}= \pm} \frac{\tilde{\Delta}_{r^{\prime}}}{\sqrt{\tilde{\omega}_{l}^{2}+\tilde{\Delta}_{r^{\prime}}^{2}}}
\end{gathered}
$$

where $\tau$ is the usual scattering time and

$$
\frac{g}{2 \pi \tau}=\sum_{r= \pm} \sum_{r^{\prime}= \pm} \int \frac{\mathrm{dS}_{r}}{(2 \pi)^{2} v_{F}^{r}} \int \frac{\mathrm{dS}_{r^{\prime}}}{(2 \pi)^{2} v_{F}^{r^{\prime}}} u_{I}^{2}\left(\mathbf{p}-\mathbf{p}^{\prime}\right) \psi_{r}(\mathbf{p}) \psi_{r^{\prime}}\left(\mathbf{p}^{\prime}\right)
$$

Here $g$ is a combined measure of the anistropy of the order parameter 35 and impurity potential. This coupling constant varies from 0 to 1 . The latter is appropriate to the case of a totally isotropic order parameter.

It follows from Eqs. (20) that intra-layer impurities enter the coupled self consistent equations via a mixture of the two band contributions. In this way they lead to pairbreaking in all instances, except for the special case of two in phase, isotropic $s$-states. Thus the $(s,-s)$ states experience a reduced $T_{c}$ in the presence of these impurities.61

We next consider inter-plane scatterers. For the sake of generality these impurities are assumed to scatter electrons within as well as between planes. The impurity Hamiltonian is given by

$$
\hat{H}_{\text {interplane }}^{\mathrm{imp}}=\sum_{m} \sum_{\mathbf{q}, \sigma}\left[u_{\|}\left(c_{1, \sigma}^{\dagger} c_{1, \sigma}+c_{2, \sigma}^{\dagger} c_{2, \sigma}\right)+u_{\perp}\left(c_{1, \sigma}^{\dagger} c_{2, \sigma}+\text { c.c. }\right)\right] e^{-\iota \mathbf{q} \cdot \mathbf{R}_{m}}
$$

It is important to note that this Hamiltonian is diagonal in the band language and that no average is taken over sites in the vertical direction. Thus the renormalized self-energy and order parameter satisfy equations which are decoupled in the band index

$$
\begin{gathered}
\tilde{\omega}_{l, \pm}=\omega_{l}+\frac{1}{2 \tau_{ \pm}} \frac{\tilde{\omega}_{l, \pm}}{\sqrt{\tilde{\omega}_{l, \pm}^{2}+\tilde{\Delta}_{ \pm}^{2}}} \\
\tilde{\Delta}_{ \pm}=\bar{\Delta}_{ \pm}+\frac{g_{ \pm}}{2 \tau_{ \pm}} \frac{\tilde{\Delta}_{ \pm}}{\sqrt{\tilde{\omega}_{l, \pm}^{2}+\tilde{\Delta}_{ \pm}^{2}}}
\end{gathered}
$$

Here care must be taken to preserve the band labels on $\tau$ and $g$. This decoupling of the two bands leads to the conclusion, stated earlier, that isotropic order parameter sets (whether in or out of phase) experience no pairbreaking from inter-layer impurities.6 
In summary, we see that one way of avoiding the strong pair-breaking generally associated with substitutions at the rare earth site between the bi-layers is to consider states with the symmetry $(s,-s)$. For this reason, along with the $\pi$ junction behavior discussed in the previous section, this state should be considered as a potentially interesting candidate for the YBCO system.

\section{FORMULATION AND ANALYSIS OF THE $N$-LAYER PROBLEM}

\section{A. Gap Equation in the Band Representation}

In this section, we treat the general $N$-layer problem. The system under consideration corresponds to a stack of decoupled $N$-layer structural units, each layer of which consists of a two dimensional copper oxide plane. In the limit of infinite $N$, we recover essentially the usual Bloch wave description of a collection of copper oxide mono-layers, aligned along the $c$-axis. 10.13 Just as in the bi-layer case, we find that there are two competing states. Depending on the relative size of the intra- and inter-plane coupling constants; one of the two is stable, while the other is metastable. These two limits correspond to inter- and intralayer dominated states. In the band language the latter are in phase and the former out of phase. Because the system contains $N$ such bands, the inter-layer gap parameters should be viewed as sinusoidally modulated with varying band index, as will be illustrated in more detail below.

Just as in the bi-layer case, the charge carriers within individual planes and on adjacent planes within a unit cell are assumed to interact via a non- retarded pair potential. Hopping of quasiparticles between adjacent planes within a unit cell is determined by the hopping matrix element $t_{\perp}$. No hopping is allowed between unit cells, as a consequence of our assumption that the $c$-axis coupling is incoherent. As before, only singlet intraband pair states are considered. Many of the detailed derivations in this section may be found in Appendix A1.

The non-interacting Hamiltonian has the form

$$
\hat{H}_{N}=\sum_{\sigma}\left[\sum_{i=1}^{N} \xi(\mathbf{q}) c_{i \sigma}^{\dagger} c_{i \sigma}-\sum_{i=1}^{N-1}\left(t_{\perp} c_{i \sigma}^{\dagger} c_{i+1, \sigma}+\text { c.c. }\right)\right]
$$

where $i$ is a layer and $\sigma$ a spin index. The superconducting order parameter is defined by

$$
\Delta_{i j}(\mathbf{q})=\sum_{\mathbf{q}^{\prime}} V_{i j}\left(\mathbf{q}, \mathbf{q}^{\prime}\right) F_{i j}\left(\mathbf{q}^{\prime}\right)
$$

where $V_{i j}$ is the interaction between electrons on layer $i$ and layer $j$ and the anomalous Green's function $F_{i j}(\mathbf{q})=\left\langle c_{i \uparrow}(\mathbf{q}) c_{j \downarrow}(-\mathbf{q})\right\rangle$ is antisymmetrized with respect to spin indices.

The anomalous component of the superconducting Green's function is obtained by performing the usual matrix inversion

$$
\mathcal{G}^{-1}=\left(\begin{array}{cc}
\iota \omega_{l}-H_{N} & -\Delta \\
-\Delta^{*} & \iota \omega_{l}+H_{N}
\end{array}\right)
$$


The entries in this matrix problem are $2 N \times 2 N$ matrices with two spin degrees of freedom and $N$ layer indices.

We diagonalize Eq. (26) in the spin degrees of freedom by multiplying the entire equation from the right by $\sigma_{2}$. The equations are then transformed to the band picture by diagonalizing the normal state Hamiltonian $H_{N}$. The resulting energy dispersion in band $r$ of the Hamiltonian is given by

$$
\epsilon_{r}(\mathbf{q})=\xi(\mathbf{q})+2 t_{\perp} \cos \left(\frac{r \pi}{N+1}\right) \quad r=1, \ldots, N
$$

We will consider only pairing of electrons within individual bands so that the order parameter is diagonal in the band representation with components $\Delta_{r}$. With a fully diagonalized Green's function the gap equation for the $N$-layer system can now be readily obtained. Upon performing the sum over Matsubara frequencies, we find

$$
\Delta_{r}(\mathbf{q})=-\sum_{\mathbf{q}^{\prime}} \sum_{r^{\prime}=1}^{N} V^{r, r^{\prime}}\left(\mathbf{q}, \mathbf{q}^{\prime}\right) \frac{\Delta_{r^{\prime}}\left(\mathbf{q}^{\prime}\right)}{2 E_{r^{\prime}}\left(\mathbf{q}^{\prime}\right)} \tanh \left[\frac{1}{2} \beta E_{r^{\prime}}\left(\mathbf{q}^{\prime}\right)\right]
$$

The energy dispersion of the elementary excitations is given for each band $r$ by the usual relation $E_{r}=\sqrt{\epsilon_{r}^{2}+\left|\Delta_{r}\right|^{2}}$. Equation (28) is the central equation of this section. In Appendix A3 we generalize this result further by extending it to an infinite stack of layers, corresponding to a fully three dimensional lattice.

\section{B. Solutions of the Gap Equation for Small $t_{\perp}$}

In this sub-section we establish the nature of the two competing states which are the stable and metastable solutions to the gap equations derived from Eq. (28)). These are most readily introduced by considering first the limit of small $t_{\perp}$. For arbitrarily small $t_{\perp}$, the two solutions become independent and appear with different onset or transition temperatures. These two states are respectively associated with pure intra- and pure interlayer pairing. Moreover, in this limit analytical results can be obtained, while the more general case of non-zero $t_{\perp}$ is treated numerically.

After some algebra, which is outlined in Appendix A2, it follows that the solution to the gap equation is given by

$$
\Delta_{r}(\mathbf{q})=\Delta_{\|, 0} \psi_{\|}(\mathbf{q})-2 \cos \left(\frac{r \pi}{N+1}\right) \Delta_{\perp, 0} \psi_{\perp}(\mathbf{q})
$$

where the two Fermi surface functions $\psi_{\|}(\mathbf{q})$ and $\psi_{\perp}(\mathbf{q})$ satisfy

$$
\begin{aligned}
\Omega_{\|} \psi_{\|}(\mathbf{q}) & =-\int \frac{\mathrm{d} S}{(2 \pi)^{2} v_{F}} V_{\|}\left(\mathbf{q}, \mathbf{q}^{\prime}\right) \psi_{\|}\left(\mathbf{q}^{\prime}\right) \\
\Omega_{\perp} \psi_{\perp}(\mathbf{q}) & =-\int \frac{\mathrm{d} S}{(2 \pi)^{2} v_{F}} V_{\perp}\left(\mathbf{q}, \mathbf{q}^{\prime}\right) \psi_{\perp}\left(\mathbf{q}^{\prime}\right)
\end{aligned}
$$

Here the integrations are over the degenerate bands of the Fermi surface and the related cnumbers, $\Omega_{\|}$and $\Omega_{\perp}$, are related to the respective transition temperatures $T_{\| / \perp, 0}$ determined by the equation 


$$
\ln \left(\frac{1.14 \omega_{c}}{k_{B} T_{\| / \perp, 0}}\right)=\frac{1}{\Omega_{\| / \perp}}
$$

Finally, the two competing states are associated with taking either one of the two parameters in $\Delta_{\|, 0}$ or $\Delta_{\perp, 0}$ in Eq. (29) to be zero.

The above results can be generalized to the case of finite $t_{\perp}$, using numerical techniques. When $t_{\perp}$ is finite, the two parameters $\Delta_{\|, 0}$ and $\Delta_{\perp, 0}$ can be simultaneously non-zero in which case both $\psi_{\|}$and $\psi_{\perp}$ will belong to the same irreducible representation of the lattice point group.

To illustrate these results, we plot the amplitudes on the various bands of the competing inter- and intralayer pair states for $N=4$ and $N=7$ in Figure 8. As in the bi-layer case, if the dominant interaction is attractive then the order parameter will be nodeless, while a repulsive interaction will yield a nodal solution with exact form determined by the details of the interaction and the band structure (as discussed in Section IIIB 1). In panels (a) and (b) of Figure 8 the magnitude of the order parameter is plotted as a function of band index $r$ for the intraband (a) and interband (b) states for a four layer system. The dotted line indicates the analytical solution for $t_{\perp}=0$ and the histogram bars illustrate the numerical results for moderate $t_{\perp}$ (comparable in magniude to the separation of the Fermi level from the van Hove points). In this way some deviation from the analytically obtained curve is seen as the solid bars differ slightly from the dotted line. It is clear that the two competing solutions represent a natural generalization of the bi-layer results to an $N$-layer system. Similar results are plotted for the seven layer system in panels (c) and (d). It follows from the figures and the above discussion that in these higher $N$ systems, even more complex behavior can be obtained, with a range of signs and magnitudes of the order parameters associated with the different bands.

To make this complexity even more explicit, we have considered the case of $N=3$ for the case of a dominant attractive interlayer interaction and a weak in-plane repulsion both peaked at $(\pi, \pi)$. In this case the hopping is slightly larger than in the previous figure. Figure 9 shows our solution to the gap equation and the normal state bandstructure (inset) for this 3 band model of orthorhombic YBCO with the two lower energy bands closed about $X$ and the highest energy band open. This last band can be viewed as simulating the chain band in $\mathrm{YBCO}, 21$ since conduction in this band is only possible along one principle axis. Solution of the 3 layer gap equation clearly shows the mixing between the two components of the solution. The middle (plane-like) band has a pure $d$-wave solution, since interlayer pairing contributes very little to the gap on this band, while the other two bands have $s$-wave symmetry. The physics of this 3 band model is equally complex. Because of the dominance of an $s$-wave order parameter component, one expects that the magnitude of $T_{c}$ is only mildly affected by impurity scatterers. On the other hand, a nodal solution on one of the bands will yield power law dependences in thermodynamic functions at low temperatures. While the above model should not be viewed as a detailed representation of YBCO, it serves to illustrate the rich array of phenomena which are associated with multiband systems. 


\section{Basis Functions and Van Hove Effects}

Band structure effects have played an important role in our analysis, particularly when the Fermi energy lies in the vicinity of the van Hove singularities. We have seen that these singularities distort the shape of the order parameter. They also play a key role in determining the relative stability of various solutions to the gap equations. In this subsection we show that, of all the different gap symmetries, two are able to take maximal advantage of the van Hove points: these are the nodeless $s$ if the interaction is attractive and the " $d_{x^{2}-y^{2}}$ " states ${ }^{3} 3$ in the case of a repulsive interaction. It should be noted that there is a considerable literature on the effect of the van Hove singularities on raising $T_{c}$. 64 In this paper we focus on the interplay of the van Hove singularity and order parameter symmetry. Earlier work 13.65 has shown that in the more general strong coupling picture, states far from the Fermi energy may wash out, to some degree, the effectiveness of the van Hove singularity in raising $T_{c}$. While here we use a weak coupling approach to address the order parameter symmetry the same qualitative behaviour can be expected to follow in more general strong coupling calculations.

To quantify the van Hove effects we study the linearized form of Equation (28)

$$
\Omega \Delta_{r}(\mathbf{q})=-\sum_{r^{\prime}=1}^{N} \int_{\epsilon_{r^{\prime}}=E_{F}} \frac{\mathrm{dS}_{r^{\prime}}}{(2 \pi)^{2} v_{F}^{r^{\prime}}\left(\mathbf{q}^{\prime}\right)} V^{r, r^{\prime}}\left(\mathbf{q}, \mathbf{q}^{\prime}\right) \Delta_{r^{\prime}}\left(\mathbf{q}^{\prime}\right)
$$

where the integrations are performed over segments of the Fermi surface corresponding to the different bands and the eigenvalue $\Omega$ defines the BCS transition temperature $T_{c}$

$$
\Omega^{-1}=\ln \left(\frac{1.14 \omega_{c}}{k_{B} T_{c}}\right)
$$

The Fermi velocity on band $r$ is $v_{F}^{r}$, and $E_{F}$ is the Fermi energy.

We define a complete set of orthonormal basis functions over the Fermi surface, 6 which are non-zero over only a single band $r$ and assume further that these $\psi_{i}^{r(\Gamma)}(\mathbf{q})$ belong to an irreducible representation $\Gamma$ of the lattice point group. These basis functions satisfy

$$
\sum_{p=1}^{N} \int_{\epsilon_{p}=E_{F}} \frac{\mathrm{dS}_{p}}{(2 \pi)^{2} v_{F}^{p}(\mathbf{q})} \psi_{i}^{r(\Gamma)}(\mathbf{q}) \psi_{j}^{r^{\prime}\left(\Gamma^{\prime}\right)}(\mathbf{q})=\delta_{i, j} \delta_{r, r^{\prime}} \delta_{\Gamma, \Gamma^{\prime}}
$$

The pair wave function and interaction potential河 are expanded in terms of these Fermi surface harmonics as

$$
\Delta_{r}=\sum_{\Gamma, i} \Delta_{i}^{r(\Gamma)} \psi_{i}^{r(\Gamma)}
$$

and

$$
V^{r, r^{\prime}}\left(\mathbf{q}, \mathbf{q}^{\prime}\right)=\sum_{\Gamma, i, j} V_{i, j}^{r, r^{\prime}(\Gamma)} \psi_{i}^{r(\Gamma)}(\mathbf{q}) \psi_{j}^{r^{\prime}(\Gamma)}\left(\mathbf{q}^{\prime}\right)
$$

The gap equation is thus reduced to the simple set of eigenvalue problems

$$
\Omega \Delta_{i}^{r(\Gamma)}=-\sum_{j, r^{\prime}} V_{i, j}^{r, r^{\prime}(\Gamma)} \Delta_{j}^{r^{\prime}(\Gamma)}
$$


By working in the space of functions defined by Eq.(34) it is clear that the basis functions

$\psi_{i}^{r(\Gamma)}$ are weighted by the inverse of $\sqrt{v_{F}^{r}}$ and so regions along the directions of the van Hove points give a correspondingly greater contribution in the gap equation. If the interaction $V^{r, r^{\prime}}\left(\mathbf{q}, \mathbf{q}^{\prime}\right)$ coupling two points on the Fermi surface is repulsive (attractive) then states with opposite (same) phase at these two points will be favoured. Thus we can conclude that the $d_{x^{2}-y^{2}}$ basis function will benefit most from the van Hove points for repulsive interactions while the nodeless $s$ function will be most enhanced by an attractive interaction.

The effect of the van Hove singularities in the single particle density of states on the superconducting order parameter can be quantified through the pairing density of states $(\mathrm{PDOS}) 6$

$$
P_{\Delta}\left(E_{F}\right)=\frac{\int \frac{\mathrm{d} S}{(2 \pi)^{2} v_{F}}|\Delta(\mathbf{q})|^{2}}{\int \mathrm{d} S|\Delta(\mathbf{q})|^{2}}
$$

The integrals are taken over all bands of the Fermi surface. To illustrate this function and its relation to the van Hove singularities, in Figure (10a) we plot $P_{\Delta}$ as a function of the Fermi energy for various solutions to the gap equation. Here we focus on the one layer case for clarity. It can be seen that a peak appears at the van Hove point for all irreducible representations. It is, however, more appropriate to normalize by the single particle density of states $P_{\Delta=1}$. This gives a more accurate indication of the degree to which the pair wave function is stabilized by the density of states. The result for the four different irreducible representations of the $D_{4 h}$ lattice of LSCO is plotted against Fermi energy in Figure (10b). Here we select that $\mathbf{Q}$ which results in a maximal $T_{c}$ for each representation. In this way, we see that the $B_{1 g}$ solution, ie. the " $d_{x^{2}-y^{2}}$ " state, and the nodeless $A_{1 g}$ "s"-wave solution indeed benefit much more from the van Hove singularity than do all other states.

In summary, there are two states which take maximal advantage of the van Hove points. These are the nodeless $s$-wave state which occurs only for attractive interactions and the $d_{x^{2}-y^{2}}$ state, appropriate to the case of repulsive interactions. It should be stressed that in general the order parameter will not have these simple functional forms corresponding to a single basis function. This is is all the more striking as the Fermi energy approaches the van Hove singularity where admixtures of higher order basis functions are most evident. While in the presence of multiple bands, the results plotted in Figure 10 become more complicated, the essential features still remain.

\section{CONCLUSION}

The most important issues in the field of high $T_{c}$ superconductivity involve determinations of the order parameter symmetry and the superconducting pairing mechanism. While there are, clearly, no definitive answers to be had at this time, this paper has been directed towards addressing these two issues. We have emphacized the role of multi-layer effects in the cuprates, in large part because the most well characterized material, YBCO, has two copper oxide planes. This complexity leads to complications in inferring the order parameter symmetry from various experimental tests. It also suggests that there are different 
(inter- and intra-plane) channels which should be considered in any microscopic theory of the pairing.

In reference to the order parameter symmetry, we have found that a multi-layer system should be characterized by distinct gaps appropriate to each of the multiple bands. A bilayer material such as YBCO has two gaps, a tri-layer, three, etc. In the presence of even a very small amount of orthorhombicity, one of the gaps can be predominantly of $d$, while another of $s$ symmetry. (Throughout this paper we refer to $d$ and $s$ states as those which are odd or even, respectively, under a $\pi / 2$ rotation of the wave-vector). One may be node-less while the other has nodes. The multiple gaps can be in or out of phase. In this way the observation of power law behavior in thermodynamics may reflect on only one of the order parameters in question. The observation of $\pi$ phase shifts in Josephson corner junction experiments on YBCO must be viewed more widely in this multi- band context. Indeed, we have found that this behavior can be associated with two out of phase $s$-states in the presence of (weak) orthorhombicity. This orthorhombicity leads to a strong asymmetry of the $s$ and $-s$ states, so that one gap function is elongated along the $a$ and the other along the $b$ axes of the crystal. The net Josephson current behaves rather similarly to a $d_{x^{2}-y^{2}}$ state, although the thermodynamical behavior need not exhibit the power laws of this state. Finally, the behavior of multi-layer systems in the presence of impurities is similarly complex. Intraand inter-layer impurities suppress $T_{c}$ in a different fashion. The $s,-s$ state is of interest because it obeys an Anderson theorem with respect to inter-layer substitutions. This may help explain why substitutions at the rare earth site in YBCO make little or no difference to the magnitude of $T_{c}$.

In the process of investigating very generic model interactions for the superconductivity, we have inferred information about microscopic constraints on the pairing mechanism. $d_{x^{2}-y^{2}}$-like states are found to be general solutions to the gap equation for repulsive interactions, in large part because they possess the fewest number of nodes and thereby the highest transition temperatures. In this way, they should not be specifically associated with a spin fluctuation driven pairing mechanism. Moreover, van Hove effects act to stabilize some order parameters over others. Of these the $d_{x^{2}-y^{2}}$-like symmetry is, again, the most notable. Orthorhombicity further enhances this stabilization. Thus for a variety of reasons, this state emerges as a natural solution to the gap equation(s) in the presence of repulsive interactions.

While we have emphacized the bi-layer $(N=2)$ case, we also presented general multilayer calculations which view the $c$-axis as consisting of decoupled structural units, each of which contains $N$ copper oxide layers. By contrast, within the unit cell the intra- and interplane hopping is appreciable and plays an important role in giving rise to $N$ distinct bands. These $N>2$ calculations may be particularly relevant in the context of the $\mathrm{Hg}$ and Bi based cuprates. In treating the superconductivity, we have included intra- and inter-plane pairing interactions in parallel with the above intra- and inter-plane hopping. We demonstrated that, regardless of the number of layers $N$ in the unit cell, there are always two competing states: one of which is intra-plane dominated, so that the resulting $N$ band- gaps are in phase, and one of which is inter-plane dominated, so that the $N$ gaps are sinusoidally modulated. Small changes in the parameterizations can lead to a transition from one of these states to another. Thus it may be inferred that the order parameter symmetry is potentially variable from one cuprate to another and from one stoichiometry to another. 
While the inclusion of these multilayer effects has been seen to introduce considerable complexity into the classification of the order parameter symmetry, this complexity is inescapable. As long as the layers communicate via one or two body processes (i.e., via hopping or pairing interactions), superconductivity in the high $T_{c}$ cuprates must include these multilayer effects.

Note Added: After this manuscript was completed we learned of recent experiments from C. Tsuei and co-workers in which $\pi$ phase shifts have been reported for a one layer, tetragonal $\mathrm{Tl}$ compound.

\section{ACKNOWLEDGMENTS}

We thank M. Norman and R. Klemm for useful conversations. This work was supported by the National Science Foundation (DMR 9120000) through the Science and Technology Centre for Superconductivity.

\section{APPENDIX A: CALCULATION OF GAP EQUATION FOR $N$-LAYERS}

\section{Derivation}

To begin we need to calculate the superconducting Green's function defined by the $2 N \times 2 N$ matrix

$$
\mathcal{G}^{-1}=\left(\begin{array}{cc}
\iota \omega_{l}-H_{N} & -\Delta \\
-\Delta^{*} & \iota \omega_{l}+H_{N}
\end{array}\right)
$$

The components $H_{N}$ and $\Delta$ are defined in Section IIIA. First we diagonalize the normal state Hamiltonian. For this purpose we define the set of characteristic polynomials

$$
D_{N}(\xi-\epsilon)=\operatorname{det}\left(H_{N}-\epsilon \mathbb{1}_{N}\right)
$$

and observe that they satisfy the recursion relation

$$
\begin{aligned}
& D_{i}(x)=x D_{i-1}(x)-t_{\perp}^{2} D_{i-2}(x) \\
& D_{0}(x)=0 \\
& D_{1}(x)=x
\end{aligned}
$$

By using the known properties of the Chebyshev polynomials it is straightforward to show that these characteristic polynomials have the form

$$
\begin{aligned}
D_{N}(x) & =\left(-t_{\perp}\right)^{N} \frac{\sin [(N+1) q]}{\sin (q)} \\
x & =-2 t_{\perp} \cos (q)
\end{aligned}
$$

From the zeroes of these polynomials we can determine the normal state energies

$$
\epsilon_{r}(\mathbf{q})=\xi(\mathbf{q})+2 t_{\perp} \cos \left(\frac{r \pi}{N+1}\right) \quad r=1, \ldots, N
$$


An orthonormal set of eigenstates of $H_{N}$ can be found similarly. The $i^{\text {th }}$ component of the state associated with band $r$ is given by

$$
\phi_{i}^{r}=\sqrt{\frac{2}{N+1}}(-1)^{i+r} \sin \left(\frac{i r \pi}{N+1}\right)
$$

and consequently the components of the unitary matrix which diagonalizes (A1) are given by

$$
U_{i j}=\sqrt{\frac{2}{N+1}}(-1)^{i+j} \sin \left(\frac{i j \pi}{N+1}\right)
$$

The particle creation operators in the band and layer language are thus related by the equation

$$
a_{i}^{\dagger}=\sum_{j=1}^{N} U_{i j} c_{j}^{\dagger}
$$

Since we predominantly focus on intraband pairing the order parameter associated with each band $r$ is thus defined through the anomalous Green's function components

$$
\begin{aligned}
F_{r, r^{\prime}}(\mathbf{q}) & =\frac{1}{\sqrt{2}}\left\langle a_{r \uparrow}(\mathbf{q}) a_{r^{\prime} \downarrow}(-\mathbf{q})-a_{r \downarrow}(\mathbf{q}) a_{r^{\prime} \uparrow}(-\mathbf{q})\right\rangle \\
& =\frac{2}{N+1} \sum_{i=1}^{N} \sum_{j=1}^{N}(-1)^{i+j} \sin \left(\frac{i r \pi}{N+1}\right) \sin \left(\frac{j r^{\prime} \pi}{N+1}\right) F_{i, j}(\mathbf{q}) \delta_{r, r^{\prime}}
\end{aligned}
$$

We thus define the $N$ diagonal components of the order parameter in the band language $\Delta_{r}$ by the equation

$$
\Delta_{r}=\frac{2}{N+1} \sum_{i=1}^{N} \sum_{j=1}^{N}(-1)^{i+j} \sin \left(\frac{i r \pi}{N+1}\right) \sin \left(\frac{j r \pi}{N+1}\right) \Delta_{i, j}
$$

With the pairing restricted to intralayer pairing and pairing between nearest neighbour planes we can use the definition of $\Delta_{i, j}$ to write the order parameter on a given band $r$ in terms of the anomalous part of the Green's function

$$
\begin{aligned}
\Delta_{r}(\mathbf{q})= & \frac{1}{N+1} \sum_{\mathbf{q}^{\prime}}\left\{\sum_{i=1}^{N} V_{\|}\left(\mathbf{q}, \mathbf{q}^{\prime}\right) \sin \left(\frac{i r \pi}{N+1}\right) \sin \left(\frac{i r^{\prime} \pi}{N+1}\right) F_{i i}\left(\mathbf{q}^{\prime}\right)\right. \\
& \left.-2 \sum_{i=1}^{N-1} V_{\perp}\left(\mathbf{q}, \mathbf{q}^{\prime}\right) \sin \left(\frac{i r \pi}{N+1}\right) \sin \left(\frac{(i+1) r \pi}{N+1}\right)\left[F_{i, i+1}\left(\mathbf{q}^{\prime}\right)+F_{i+1, i}\left(\mathbf{q}^{\prime}\right)\right]\right\}
\end{aligned}
$$

With a fully diagonalized Green's function the gap equation for the $N$-layer system can now be readily obtained. Upon performing the sum over Matsubara frequencies in the usual way we get

$$
\Delta_{r}(\mathbf{q})=-\sum_{\mathbf{q}^{\prime}} \sum_{r^{\prime}=1}^{N} V^{r, r^{\prime}}\left(\mathbf{q}, \mathbf{q}^{\prime}\right) \frac{\Delta_{r^{\prime}}\left(\mathbf{q}^{\prime}\right)}{2 E_{r^{\prime}}\left(\mathbf{q}^{\prime}\right)} \tanh \left[\frac{1}{2} \beta E_{r^{\prime}}\left(\mathbf{q}^{\prime}\right)\right]
$$


where the interaction in the band representation is defined as

$$
\begin{gathered}
V^{r, r^{\prime}}\left(\mathbf{q}, \mathbf{q}^{\prime}\right)=\frac{1}{N+1}\left[\left(1+\frac{1}{2} \theta_{r, r^{\prime}}^{+(N)}\right) V_{\|}\left(\mathbf{q}, \mathbf{q}^{\prime}\right)+\left(2 \cos \left(\frac{r \pi}{N+1}\right) \cos \left(\frac{r^{\prime} \pi}{N+1}\right)+\theta_{r, r^{\prime}}^{-(N)}\right) V_{\perp}\left(\mathbf{q}, \mathbf{q}^{\prime}\right)\right] \\
\theta_{r, r^{\prime}}^{ \pm(N)}=\delta_{r, r^{\prime}} \pm \delta_{r+r^{\prime}, N+1}
\end{gathered}
$$

where the quasi-particle dispersions in the superconducting state are defined by the usual relation $E_{r}=\sqrt{\epsilon_{r}^{2}+\left|\Delta_{r}\right|^{2}}$.

\section{The Small $t_{\perp}$ Limit}

For infinitesimal $t_{\perp}$ it is interesting to transform Eq. (A12) to the layer representation using Eq. (A10). Then to zeroth order in $t_{\perp}$ the $N$ intralayer problems uncouple from the $N-1$ interlayer problems yielding the following two sets of gap equations

$$
\begin{aligned}
\Delta_{i, i}(\mathbf{q}) & =\frac{-1}{N+1} \sum_{\mathbf{q}^{\prime}} V_{\|}\left(\mathbf{q}, \mathbf{q}^{\prime}\right) \frac{\tanh \left[\frac{1}{2} \beta E\left(\mathbf{q}^{\prime}\right)\right]}{2 E\left(\mathbf{q}^{\prime}\right)} \sum_{j=1}^{N}\left(1+\frac{1}{2} \theta_{i, j}^{+(N)}\right) \Delta_{j, j}\left(\mathbf{q}^{\prime}\right) \\
\Delta_{i, i+1}(\mathbf{q}) & =\frac{-1}{N+1} \sum_{\mathbf{q}^{\prime}} V_{\perp}\left(\mathbf{q}, \mathbf{q}^{\prime}\right) \frac{\tanh \left[\frac{1}{2} \beta E\left(\mathbf{q}^{\prime}\right)\right]}{2 E\left(\mathbf{q}^{\prime}\right)} \sum_{j=1}^{N-1}\left(1+\theta_{i, j}^{+(N-1)}\right) \Delta_{j, j+1}\left(\mathbf{q}^{\prime}\right)
\end{aligned}
$$

Linearizing with respect to $\Delta$ and solving for the eigenvalues and eigenfunctions of these two equations gives the $T_{c}$ 's of the various pairing states available to the system and only one of these solutions will correspond to the maximal value of $T_{c}$.

Let us first define two functions on the fermi-surface, $\psi_{\|}(\mathbf{q})$ and $\psi_{\perp}(\mathbf{q})$, and two numbers, $\Omega_{\|}$and $\Omega_{\perp}$, which satisfy the two equations

$$
\begin{aligned}
\Omega_{\|} \psi_{\|}(\mathbf{q}) & =-\int \frac{\mathrm{d} S}{(2 \pi)^{2} v_{F}} V_{\|}\left(\mathbf{q}, \mathbf{q}^{\prime}\right) \psi_{\|}\left(\mathbf{q}^{\prime}\right) \\
\Omega_{\perp} \psi_{\perp}(\mathbf{q}) & =-\int \frac{\mathrm{d} S}{(2 \pi)^{2} v_{F}} V_{\perp}\left(\mathbf{q}, \mathbf{q}^{\prime}\right) \psi_{\perp}\left(\mathbf{q}^{\prime}\right)
\end{aligned}
$$

The integrations are over the degenerate bands of the Fermi surface. The pair amplitudes can be written in the form

$$
\begin{aligned}
\Delta_{i, i}(\mathbf{q}) & =\Delta_{\|}^{i} \psi_{\|}(\mathbf{q}) \\
\Delta_{i, i+1}(\mathbf{q}) & =\Delta_{\perp}^{i} \psi_{\perp}(\mathbf{q})
\end{aligned}
$$

The gap equations (A14) are transformed in the usual manner by separating the sum over quasi-momenta to separate integrals over energy and the Fermi surface. The energy integral can then be performed to obtain a BCS-like transition temperature and using Eqs. A15 we derive the linear matrix equations

$$
\begin{gathered}
\Delta_{\|}^{i}=\ln \left(\frac{\gamma \omega_{c}}{k_{B} T_{c}}\right) \frac{\Omega_{\|}}{N+1} \sum_{j=1}^{N}\left(1+\frac{1}{2} \theta_{i, j}^{+(N)}\right) \Delta_{\|}^{j} \\
\Delta_{\perp}^{i}=\ln \left(\frac{\gamma \omega_{c}}{k_{B} T_{c}}\right) \frac{\Omega_{\perp}}{N+1} \sum_{j=1}^{N-1}\left(1+\theta_{i, j}^{+(N-1)}\right) \Delta_{\perp}^{j}
\end{gathered}
$$


where $\gamma \approx 1.14$ and $\omega_{c}$ is the usual cutoff energy. We can now characterize all the available pairing states with functional form given by $\psi_{\|}$and $\psi_{\perp}$ along with their BCS transition temperatures.

Eqs. (A17) each have one isotropic eigenvector given by $\Delta_{\| / \perp}^{i}=\Delta_{\| / \perp, 0}$ with $T_{c, 0}$ determined by the equation

$$
\ln \left(\frac{\gamma \omega_{c}}{k_{B} T_{c, 0}}\right)=\frac{1}{\Omega_{\| / \perp}}
$$

which give the most stable candidate pairing states for the two mechanisms. The remaining eigenvectors correspond either to metastable states which are symmetric in the layer index such that $\sum_{i} \Delta_{\| / \perp}^{i}=0$ with $T_{c}$ given by

$$
\ln \left(\frac{T_{c}}{T_{c, 0}}\right)=\left\{\begin{array}{c}
-\frac{N}{\Omega_{\|}} \\
-\frac{N-1}{2 \Omega_{\perp}}
\end{array}\right.
$$

or non-pairing states $\left(T_{c}=0\right)$ which are odd in the layer index.

Transforming to the band picture using Eq. (A10) we find that the intralayer pairing states are even under the transformation $r \rightarrow N+1-r$ whereas the interlayer pairing states are odd under this transformation. The two most stable candidate states thus give a superconducting order parameter of the form

$$
\Delta_{r}(\mathbf{q})=\Delta_{\|, 0} \psi_{\|}(\mathbf{q})-2 \cos \left(\frac{r \pi}{N+1}\right) \Delta_{\perp, 0} \psi_{\perp}(\mathbf{q})
$$

The two parameters $\Delta_{\|, 0}$ and $\Delta_{\perp, 0}$ are both nonzero only when $t_{\perp}$ is finite and when $\psi_{\|}$ and $\psi_{\perp}$ belong to the same irreducible representation. Since the two different pairing mechanisms give solutions of different $r$ dependence, the dominant type of pairing can be easily determined even when $t_{\perp}$ is finite. Mixing of solutions at finite $t_{\perp}$ and the effect on the transition temperature is discussed in Appendix B in the bi-layer context.

\section{Formulation In Terms of Bloch Waves}

For completeness we conclude by relating the above formulation to the usual treatment of layered materials. It would be natural to define a $z$-component of the quasi-momentum vector to be

$$
q_{z}=\frac{r \pi}{N+1}
$$

Note, however, that there are $N$ linearly independent, non-degenerate eigenstates for $0<q_{z}<\pi$ and so taking $q_{z} \rightarrow-q_{z}$ gives no new states. Thus interpreting $q_{z}$ as a momentum is rather unnatural.

The usual procedure in the case of large $N$, 10 . 3 however, is to assume periodic boundary conditions. This means that we have hopping between layers 1 and $N$ and that the system is translationally invariant along the $c$-axis. The eigenstates of this new Hamiltonian are 


$$
\phi_{j}^{r}=\frac{1}{\sqrt{N}} \exp \left(\iota \frac{2 r \pi}{N} j\right)
$$

with energies

$$
\epsilon_{r}=\xi+2 t_{\perp} \cos \left(\frac{2 r \pi}{N}\right)
$$

Taking the limit of $N$ going to infinity one obtains the gap equation for a fully three dimensional system. Again assuming only intraband pairing the gap equation becomes

$$
\Delta(\mathbf{q})=-\sum_{\mathbf{q}^{\prime}} V\left(\mathbf{q}, \mathbf{q}^{\prime}\right) \frac{\Delta\left(\mathbf{q}^{\prime}\right)}{2 E\left(\mathbf{q}^{\prime}\right)} \tanh \left[\frac{1}{2} \beta E\left(\mathbf{q}^{\prime}\right)\right]
$$

with the interaction

$$
V\left(\mathbf{q}, \mathbf{q}^{\prime}\right)=\frac{1}{N}\left[V_{\|}\left(\mathbf{q}, \mathbf{q}^{\prime}\right)+2 \cos \left(q_{z}-q_{z}^{\prime}\right) V_{\perp}\left(\mathbf{q}, \mathbf{q}^{\prime}\right)\right]
$$

and the usual quasi-particle energies $E(\mathbf{q})=\sqrt{\epsilon(\mathbf{q})^{2}+|\Delta(\mathbf{q})|^{2}}$. In the large $N$ limit this system behaves identically to the one considered throughout this paper when only singlet pairing is considered. On the other hand, it is obvious that this formulation gives very different results in the small $N$ limit. One might expect that in any real system the hopping between unit cells is different from that within a unit cell and so a more general formulation than either of these would be required. Such a formulation would, however, yield a continuous set of fermi levels within some small band and this result would be in contradiction to experimental observations.

\section{APPENDIX B: INTERBAND PAIRING}

To conclude this discussion we present a more careful treatment of the case for which $N=2$ admitting the possibility of pairing of electrons on different sub-bands of the Fermisurface. We will restrict our attention to states which are even under inversion, neglecting the possibility of a $p$-wave order parameter. This necessitates the consideration of a triplet interlayer pairing state. The normal state Hamiltonian has the form

$$
H_{2}=\left(\begin{array}{cc}
\xi & -t_{\perp} \\
-t_{\perp}^{*} & \xi
\end{array}\right)
$$

where $t_{\perp}=\left|t_{\perp}\right| e^{-\iota \varphi}$. The order parameter has the form

$$
\Delta=\left(\begin{array}{cc}
\Delta_{\|}^{e}+\Delta_{\|}^{o} & \Delta_{\perp}^{s}-\iota \sum_{i=1}^{3} \Delta_{\perp}^{t, i} \sigma_{i} \\
\Delta_{\perp}^{s}+\iota \sum_{i=1}^{3} \Delta_{\perp}^{t, i} \sigma_{i} & \Delta_{\|}^{e}-\Delta_{\|}^{o}
\end{array}\right)
$$

The $\sigma_{i}$ are the three Pauli spin matrices. Upon diagonalizing (B1) we obtain

$$
\begin{aligned}
H_{2} & =\left(\begin{array}{cc}
\xi-\left|t_{\perp}\right| & 0 \\
0 & \xi+\left|t_{\perp}\right|
\end{array}\right) \\
\Delta & =\left(\begin{array}{cc}
\Delta_{+} & \Delta_{1}-\iota \Delta_{2} \\
\Delta_{1}+\iota \Delta_{2} & \Delta_{-}
\end{array}\right)
\end{aligned}
$$


We assume that the triplet component is describable by a single complex parameter $\Delta_{\perp}^{t}$ and a real unit vector in spin space $\hat{n}$ so that $\Delta_{\perp}^{t, i}=\Delta_{\perp}^{t} \hat{n}_{i}$. The Green's function can thus be diagonalized in its spin degrees of freedom. There are, therefore, four independent parameters which describe the superconducting state. The components of the order parameter in the band representation are

$$
\begin{aligned}
\Delta_{+} & =\Delta_{\|}^{e}+\Delta_{\perp}^{s} \\
\Delta_{-} & =\Delta_{\|}^{e}-\Delta_{\perp}^{s} \\
\Delta_{1} & =\Delta_{\|}^{o} \\
\Delta_{2} & =-\Delta_{\perp}^{t} \sigma_{3}
\end{aligned}
$$

Designating the bands by $\epsilon_{ \pm}=\xi \mp\left|t_{\perp}\right|$ we solve for the anomalous parts of the Green's function as before. These may be linearized in $\Delta$ at $T_{c}$ and upon performing the sum over Matsubara frequencies we arrive at the following four gap equations

$$
\begin{aligned}
\Delta_{+}+\Delta_{-} & =-\sum_{\mathbf{q}} V_{\|}\left[\Delta_{+} \frac{\tanh \left(\frac{1}{2} \beta \epsilon_{+}\right)}{2 \epsilon_{+}}+\Delta_{-} \frac{\tanh \left(\frac{1}{2} \beta \epsilon_{-}\right)}{2 \epsilon_{-}}\right] \\
\Delta_{+}-\Delta_{-} & =-\sum_{\mathbf{q}} V_{\perp}\left[\Delta_{+} \frac{\tanh \left(\frac{1}{2} \beta \epsilon_{+}\right)}{2 \epsilon_{+}}-\Delta_{-} \frac{\tanh \left(\frac{1}{2} \beta \epsilon_{-}\right)}{2 \epsilon_{-}}\right] \\
\Delta_{1} & =-\sum_{\mathbf{q}} V_{\|} \Delta_{1} \frac{\tanh \left(\frac{1}{2} \beta \epsilon_{+}\right)+\tanh \left(\frac{1}{2} \beta \epsilon_{-}\right)}{2 \epsilon_{+}+2 \epsilon_{-}} \\
\Delta_{2} & =-\sum_{\mathbf{q}} V_{\perp} \Delta_{2} \frac{\tanh \left(\frac{1}{2} \beta \epsilon_{+}\right)+\tanh \left(\frac{1}{2} \beta \epsilon_{-}\right)}{2 \epsilon_{+}+2 \epsilon_{-}}
\end{aligned}
$$

If $t_{\perp}=0$ then there is a single transition temperature associated with the two intralayer pairing states and another transition temperature associated with the two interlayer states. Denote the larger one of these by $T_{c, 0}$. It can be shown that for non-zero $t_{\perp}$ the transitions described by Eqs. (B60) and (B6d) have a lower $T_{c}$ than the intraband pairing state transition in Eqs. (B6a) and (B6b) where for small $t_{\perp}$ the new transition of the interband pairing state $e^{69}$ with higher $T_{c}$ is given in terms of $T_{c, 0}$ by

$$
\ln \left(\frac{T_{c}}{T_{c, 0}}\right)=-0.2123\left(\frac{t_{\perp}}{k_{B} T_{c}}\right)^{2}
$$

The transition temperature for the intraband pairing states is given by

$$
\ln \left(\frac{T_{c}}{T_{c, 0}}\right)=\frac{1}{\left|\ln \frac{T_{c, \|} \mid}{T_{c, \perp}}\right|}\left(\frac{t_{\perp} R}{2 k_{B} T_{c}}\right)^{2}
$$

where the overlap between the two pair wavefunctions is

$$
R=\int \frac{\mathrm{dS}}{(2 \pi)^{2} v_{F}} \psi_{\|} \psi_{\perp}
$$

We thus see that in the presence of interlayer tunneling the favoured pairing state is always an intraband pairing state. 


\section{REFERENCES}

${ }^{1}$ D. J. Scalapino, Phys. Rep. 250, 329 (1995).

${ }^{2}$ R. C. Dynes, Sol. St. Comm. 92, 53 (1994).

${ }^{3}$ K. Levin et al., Journal of Superconductivity 7, 563 (1994).

${ }^{4}$ Z.-X. Shen et al., Phys. Rev. Lett. 70, 1553 (1993).

${ }^{5}$ M. R. Norman et al., see also Ding et al., Phys. Rev. Lett. 75, 1425 (1995). (unpublished).

${ }^{6}$ J. C. Campuzano et al., Phys. Rev. Lett. 64, 2308 (1990).

${ }^{7} \mathrm{H}$. Ding et al., the conclusions reached here regarding the absence of bilayer splitting disagree with those of Ref. 4 . (unpublished).

${ }^{8}$ D. Z. Liu, K. Levin, and J. Maly, Phys. Rev. B 51, 8680 (1995).

${ }^{9}$ A. I. Liechtenstein, I. I. Mazin, and O. Andersen, Phys. Rev. Lett. 74, 2303 (1995).

${ }^{10}$ R. A. Klemm and S. H. Liu, Phys. Rev. B 44, 7526 (1991).

${ }^{11}$ N. Bulut, D. J. Scalapino, and R. T. Scalettar, Phys. Rev. B 45, 5577 (1992).

${ }^{12}$ L. N. Bulaevskii and M. V. Zyskin, Phys. Rev. B 42, 10230 (1990).

${ }^{13}$ S. Kettemann and K. B. Efetov, Phys. Rev. B 46, 8515 (1992).

${ }^{14}$ C. C. Tsuei et al., Phys. Rev. Lett. 73, 593 (1994).

${ }^{15}$ D. A. Brawner and H. R. Ott, Phys. Rev. B 50, 6530 (1994).

${ }^{16}$ D. A. Wollman et al., Phys. Rev. Lett. 71, 2134 (1993).

${ }^{17}$ A. Mathai et al., Phys. Rev. Lett. 74, 4523 (1995).

${ }^{18}$ C. C. Tsuei, D. M. Newns, J. R. Kirtley, and P. C. Pattnaik, 1995, to be published. These authors have reached a similar conclusion.

${ }^{19}$ Q. P. Li, B. E. C. Koltenbah, and R. Joynt, Phys. Rev. B 48, 437 (1993).

${ }^{20}$ K. Kobuki and P. A. Lee (unpublished).

${ }^{21}$ I. I. Mazin, A. A. Golubov, and A. D. Zaikin (unpublished).

${ }^{22}$ V. L. Pokrovski (unpublished).

${ }^{23}$ R. Combescot and X. Leyronas (unpublished).

${ }^{24}$ S. L. Cooper and K. E. Gray, in Physical Properties of High-Temperature Superconductors $I V$, edited by D. M. Ginsberg (World Scientific, Singapore, 1994).

${ }^{25}$ P. W. Anderson, Phys. Rev. Lett. 67, 660 (1991).

${ }^{26}$ A. G. Rojo and K. Levin, Phys. Rev. B 48, 16861 (1993).

${ }^{27}$ K. B. Efetov and A. I.Larkin, Sov.Phys.-JETP 41, 76 (1975).

${ }^{28}$ S. Chakravarty and P. W. Anderson, Phys. Rev. Lett. 72, 3858 (1994).

29 J. Rossat-Mignon et al., Physica C 185, 86 (1991).

30 J. M. Tranquada et al., Phys. Rev. B 46, 5561 (1992).

${ }^{31}$ H. Mook et al., Phys. Rev. Lett. 70, 3490 (1993).

${ }^{32}$ Y. Zha, Q. Si, and K. Levin, Physica C 212, 418 (1993).

${ }^{33}$ D. Z. Liu, Y. Zha, and K. Levin (unpublished).

${ }^{34}$ D. Pines and P. Monthoux, in The Proceedings of the Stanford Conference on Spectroscopy in Novel Superconductors, J. Phys. and Chem. of Solids (Pergamon Press, New York, 1995), to be published.

35 A. J. Millis, S. Sachdev, and C. M. Varma, Phys. Rev. B 37, 4975 (1988).

${ }^{36}$ R. J. Radke, K. Levin, H.-B. Schüttler, and M. R. Norman, Phys. Rev. B 48, 653 (1993).

${ }^{37}$ A. G. Sun et al., Phys. Rev. B 50, 3266 (1994).

${ }^{38}$ O. K. Andersen and et al., Phys. Rev. B 49, 4145 (1994).

${ }^{39}$ A. A. Abrikosov, Physica C 244, 243 (1995). 
${ }^{40}$ C. M. Varma, in The Proceedings of the Stanford Conference on Spectroscopy in Novel Superconductors, J. Phys. and Chem. of Solids (Pergamon Press, New York, 1995), to be published.

${ }^{41}$ In BSCCO, superlattice effects result in two non-equivalent $\mathrm{Cu}$ sites. Thus the unit cell is face-centred orthorhombic and double the size of the YBCO unit cell with the orthorhombic axes rotated by $45^{\circ}$. For simplicity, we simulate this lattice by a unit cell which has "rhombohedral" symmetry, with a unit cell equivalent to that in YBCO, but with rotated axes of symmetry.

${ }^{42}$ H. Fukushima et al., Physica C 159, 777 (1989), these authors noted that upon addition of $\mathrm{Pb}$, BSCCO acquired a mild rhombohedral distortion.

${ }^{43}$ R. J. Radke and M. R. Norman, Phys. Rev. B 50, 9554 (1994), we use the YBCO(1,ext) band structure in the case of YBCO.

${ }^{44}$ D. Sahu, A. Langner, and T. F. George, Phys. Rev. B 38, 2466 (1988).

${ }^{45}$ M. U. Ubbens and P. A. Lee, Phys. Rev. B 50, 438 (1994).

${ }^{46}$ B. Normand, H. Kohno, and H. Fukuyama (unpublished).

${ }^{47}$ Since the system is assumed orthorhombic all states are $s-d$ mixed states; however, the order parameter on a given band will have same (opposite) sign unde $\pi / 2$ rotation if the $s(d)$ component dominates.

${ }^{48}$ A. G. Sun, D. A. Gajewski, M. B. Maple, and R. C. Dynes, Phys. Rev. Lett. 72, 2267 (1994).

${ }^{49}$ C. O'Donovan, D. Branch, J. P. Carbotte, and J. Preston, Phys. Rev. B 51, 6588 (1995).

${ }^{50}$ V. Ambegaokar and A. Baratoff, Phys. Rev. Lett. 10, 486 (1963).

${ }^{51}$ E. L. Wolf, Principles of Electron Tunneling Spectroscopy (Oxford University Press, New York, 1985).

${ }^{52}$ L. J. Buchholtz, M. Plumbo, D. Rainer, and J. A. Sauls (unpublished).

${ }^{53}$ This effect is illustrated in Figure 5 .

${ }^{54}$ T. M. Rice, private communication.

${ }^{55}$ V. K. Vlasko-Vlasov et al., Phys. Rev. Lett. 72, 3246 (1994).

${ }^{56}$ It should be noted that there are two more general coupling terms which could, in principle, be added to the quartic contribution 4 . 0 . terms do not appear in mean field theory when only pairing of electrons in a single band are considered. The analogous terms in the layer language however are present.

${ }^{57}$ When $t_{\perp}$ is small $\gamma^{\prime}$ is $\frac{0.8525}{\left(k_{B} T_{c}\right)^{2}}$ where $T_{c}$ is the larger of the two transition temperatures and $\beta^{\prime}=4 \gamma^{\prime}$.

${ }^{58}$ If $\delta=0$ then the sign is undetermined.

${ }^{59}$ R. A. Klemm and K. Scharnberg, Phys. Rev. B 24, 6361 (1981).

${ }^{60}$ K. Maki, in Superconductivity, edited by R. D. Parks (Marcel Dekker, New York, 1969), Vol. 2, Chap. 18.

${ }^{61}$ Calculating the suppression $T_{c}$ yields a result similar to that of Kettemann and Efetov 1 .

${ }^{62}$ Finding the suppression of $T_{c}$ gives a straightforward extension of the usual result 3 . Furthermore one finds that if the impurity preferentially scatters electrons on one band, then the pairing amplitude is increased on the other band.

${ }^{63}$ C. C. Tsuei, D. M. Newns, J. R. Kirtley, and P. C. Pittnaik (unpublished).

${ }^{64}$ D. M. Newns, C. C. Tsuei, P. C. Pittnaik, and C. I. Kane, Cond. Mat. Phys. 15, 273 (1992). 
${ }^{65}$ R. J. Radke, K. Levin, H.-B. Schüttler, and M. R. Norman, Phys. Rev. B 48, 15957 (1993).

${ }^{66}$ P. B. Allen, Phys. Rev. B 13, 1416 (1976).

${ }^{67}$ K. Aoi and J. C. Swihart, Phys. Rev. B 7, 1240 (1972).

${ }^{68}$ R. Fehrenbacher and M.R.Norman, Phys. Rev. Lett. 74, 3884 (1995).

${ }^{69}$ Note that for interband pairing to occur we require $\left|t_{\perp}\right|<\omega_{c}$ since only pairing between time reversed states is being considered.

${ }^{70}$ M. Sigrist and T. M. Rice, Z.Phys.B.-Condensed Matter 68, 9 (1987). 


\section{TABLES}

\begin{tabular}{crrr}
\hline \hline$\eta_{i}(\mathbf{q})$ & & \multicolumn{1}{c}{$t_{i}$} \\
& LSCO & YBCO & BSCCO \\
\hline 1 & 0 & 0 & 0.1305 \\
$\frac{1}{2}\left(\cos q_{x}+\cos q_{y}\right)$ & -1.0 & -0.50 & -0.5951 \\
$\cos q_{x} \cos q_{y}$ & 0.2 & 0.15 & 0.1636 \\
$\frac{1}{2}\left(\cos 2 q_{x}+\cos 2 q_{y}\right)$ & 0 & -0.05 & -0.0519 \\
$\frac{1}{2}\left(\cos 2 q_{x} \cos q_{y}+\cos q_{x} \cos 2 q_{y}\right)$ & 0 & 0 & -0.1117 \\
$\cos 2 q_{x} \cos 2 q_{y}$ & 0 & 0 & 0.0510 \\
$\frac{1}{2}\left(\cos q_{x}-\cos q_{y}\right)$ & 0 & 0.04 & 0 \\
$\sin q_{x} \sin q_{y}$ & 0 & 0 & 0.08 \\
\hline \hline
\end{tabular}

TABLE I. Tight binding basis functions and hopping parameters (in eV) used in numerical calculations. 


\section{FIGURES}

FIG. 1. Phase diagram showing order parameter symmetry in LSCO: (a) Near van Hove points; (b) Away from van Hove points. The two axes represent the wave vectors $Q_{x}, Q_{y}$ at which the pairing interaction has a maximum.

FIG. 2. Phase diagram for one layer model of YBCO: (a) Near van Hove points; (b) Away from van Hove points. The dotted line separates $A_{1 g}$ representations with and without $a, b$-axis $\pi$ phase shifts.

FIG. 3. Phase diagram for one layer model of BSCCO: (a) Near van Hove points; (b) Away from van Hove points. A dotted line separates states with four and eight lobes within a representation.

FIG. 4. Phase diagram for stability of $\pi$ phase shifts on the $a$ relative to the $b$ axis, in the order parameter (below solid line) Model is for YBCO with an attractive interlayer and repulsive intralayer interaction, both peaked at $\mathbf{Q}_{\mathrm{AF}}$. Hole doping fraction appears on the ordinate and relative strength of the two interactions defines the abscissa. The shaded regions denote states where the order parameter on one (light) or both (dark) bands is nodeless.

FIG. 5. Evolution of order parameter solutions as interlayer correlations are increased. The states correspond to the solutions obtained in the previous figure for $x=0.25$ and the indicated $\lambda_{\perp} / \lambda_{\|}$. Observe that proximity to the van Hove singularity results in considerable $a, b$-axis anisotropy despite the very small orthorhombicity.

FIG. 6. Schematic illustration of Josephson coupling between the anti-bonding band in YBCO (left) and a $\mathrm{Pb}$ counterelectrode (right). Overlap of the single particle wave functions is nonzero unless the two wavefunctions are perfectly aligned in the directions parallel to the plane of the interface.

FIG. 7. Possible scenarios for order parameter behavior across twin boundaries showing $\pi$ phase shifted $(d, d)$-type (a) or $(s,-s)$-type (b and c) solutions. The scenarios depicted in (a) and (c) give $\pi$ junction behaviour in $a, b$-axis corner junctions while case (b) will lead to cancellation of the $\pi$ phase shift after averaging over twin domains.

FIG. 8. Amplitude of pure intralayer and interlayer pairing solutions in the band representation for $N=4$ (a and b respectively ) and $N=7$ (c and d respectively) cases. The ideal solutions $\left(t_{\perp}=0\right)$ are denoted by the dotted lines and the actual solutions are calculated for moderately large $t_{\perp}$.

FIG. 9. Solution for a three band problem illustrating co-existence of solutions of different symmetries. The inset plots the associated band structure. 
FIG. 10. Pairing Density of States (PDOS) of the solution with highest $T_{c}$ in each irreducible representation for LSCO. Panel (a) shows the absolute PDOS in arbitrary units and panel (b) illustrates the PDOS normalized by the single particle density of states. The isotropic $s$-state (solid line) was calculated for an attractive pair interaction peaked at $(\pi, \pi)$ while the other solutions were calculated for repulsive interactions, with $\mathbf{Q}$ chosen so as to maximize $T_{c}$. 


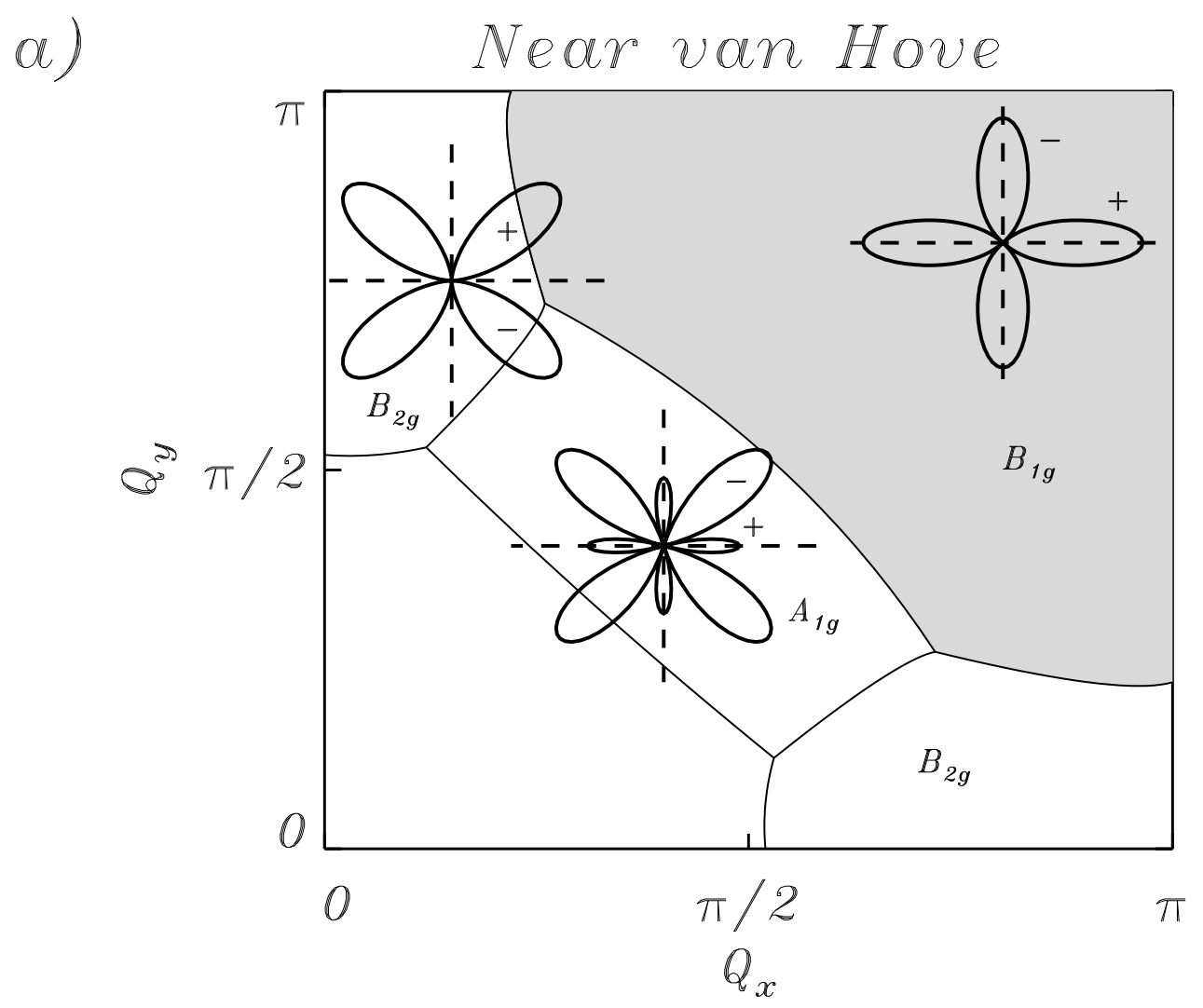

b)

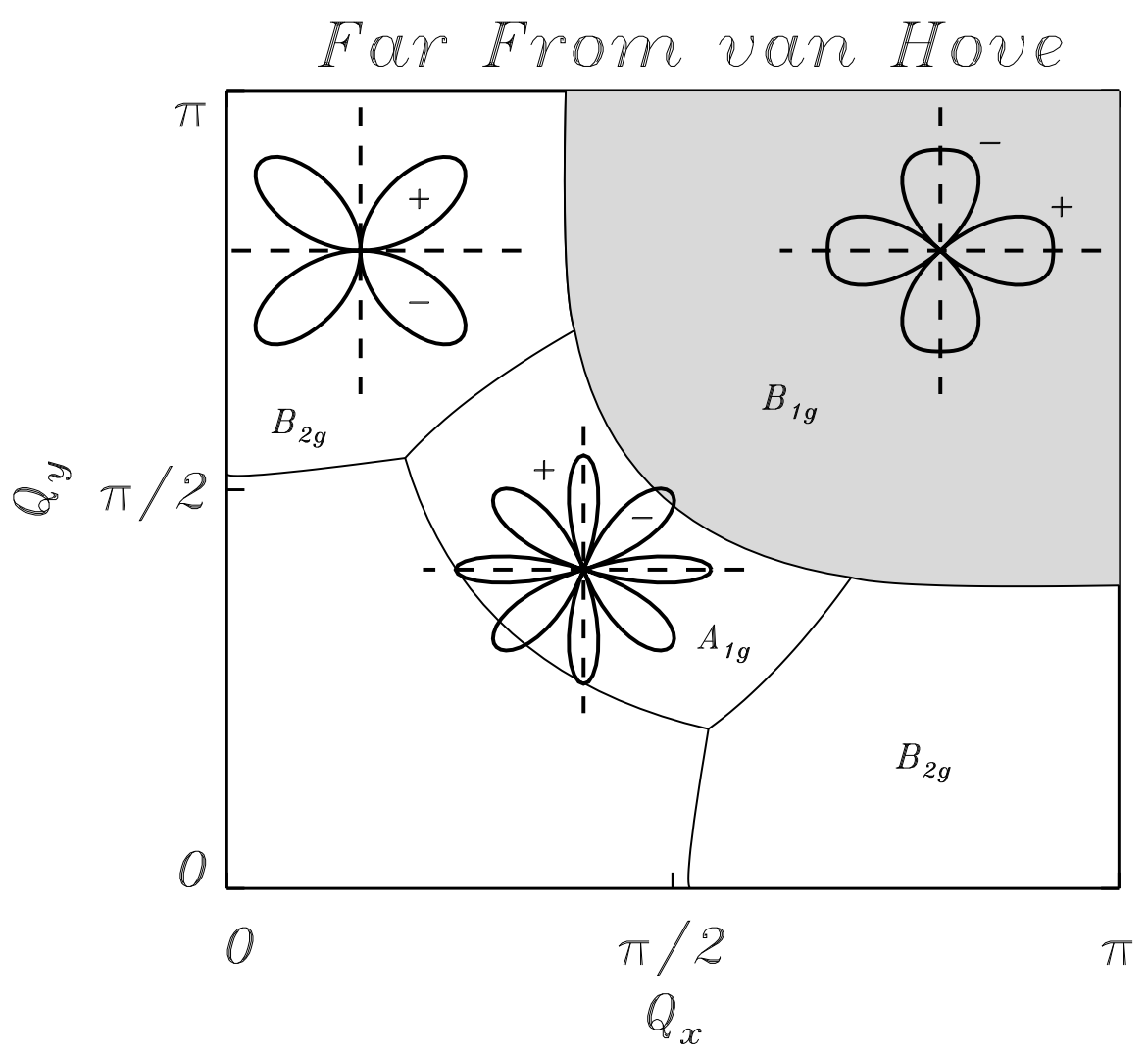




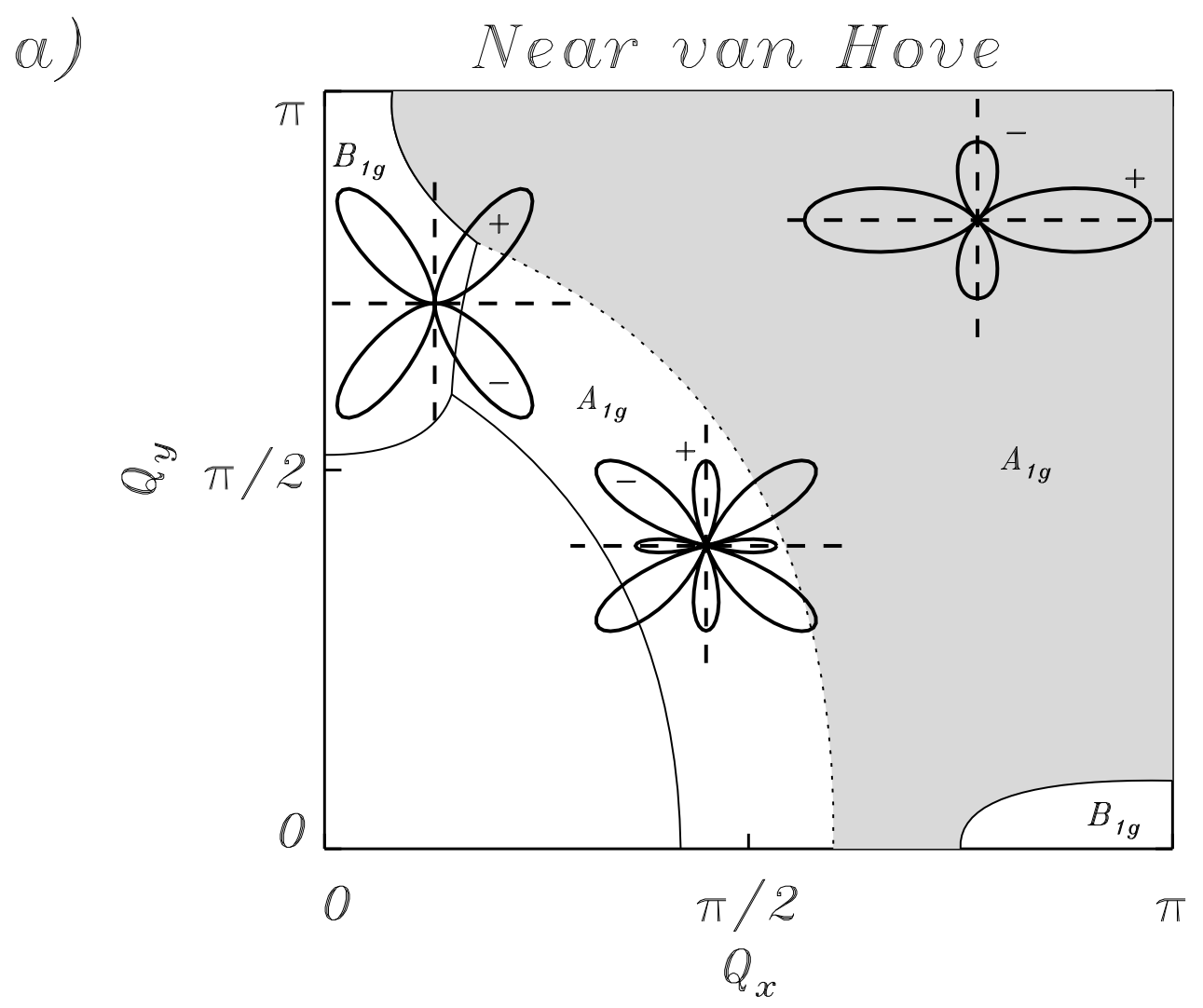

b)

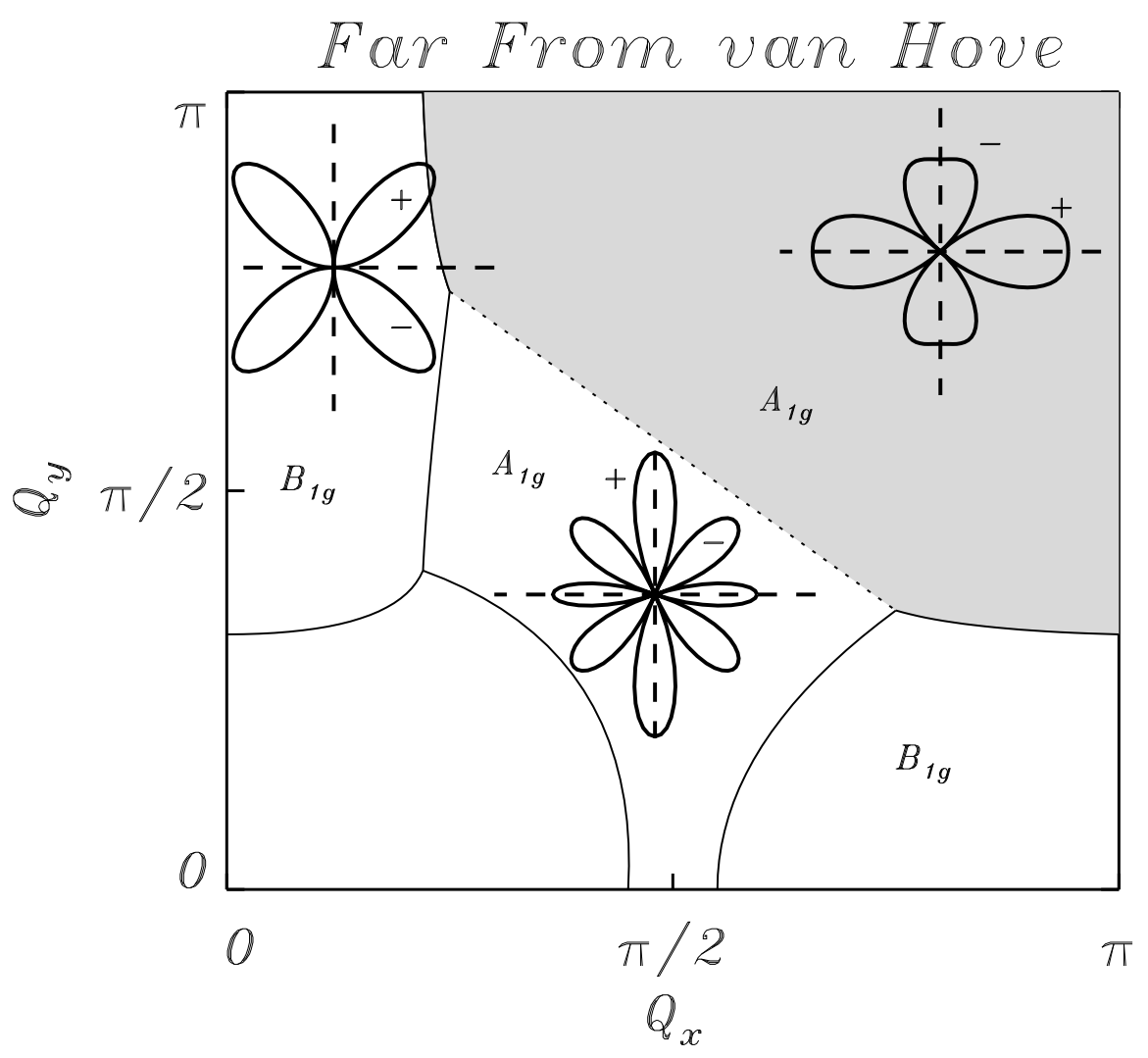




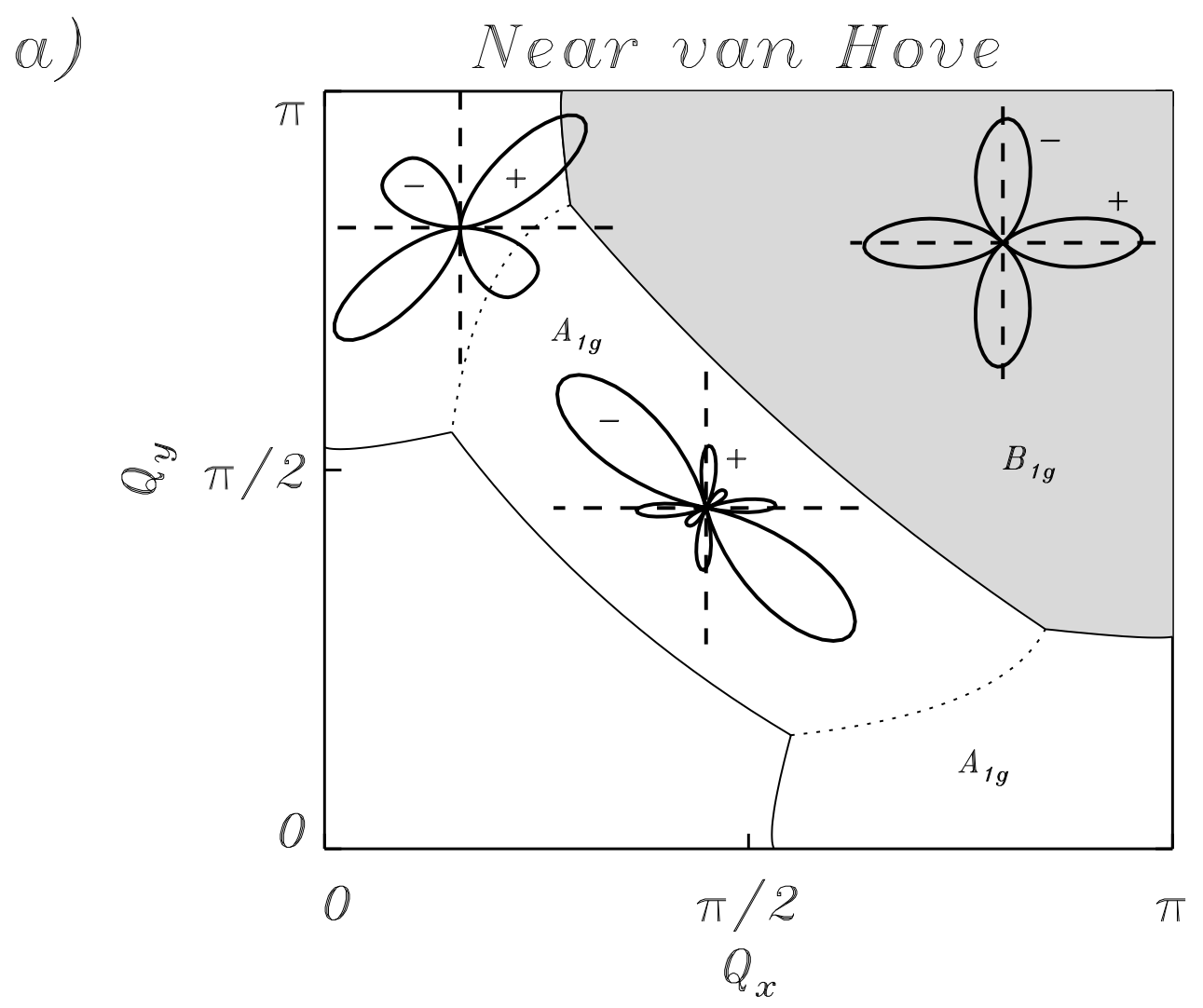

b)

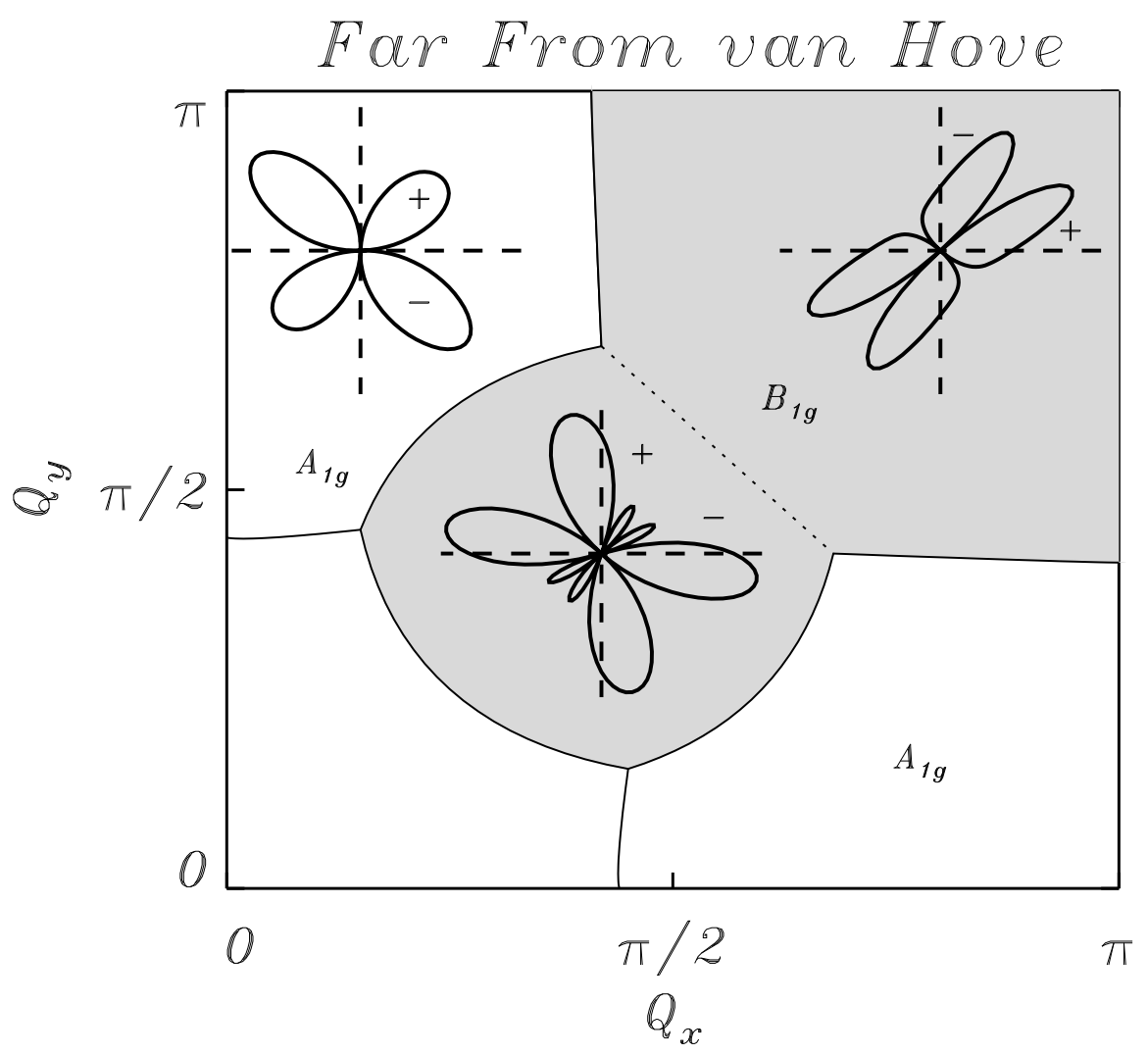




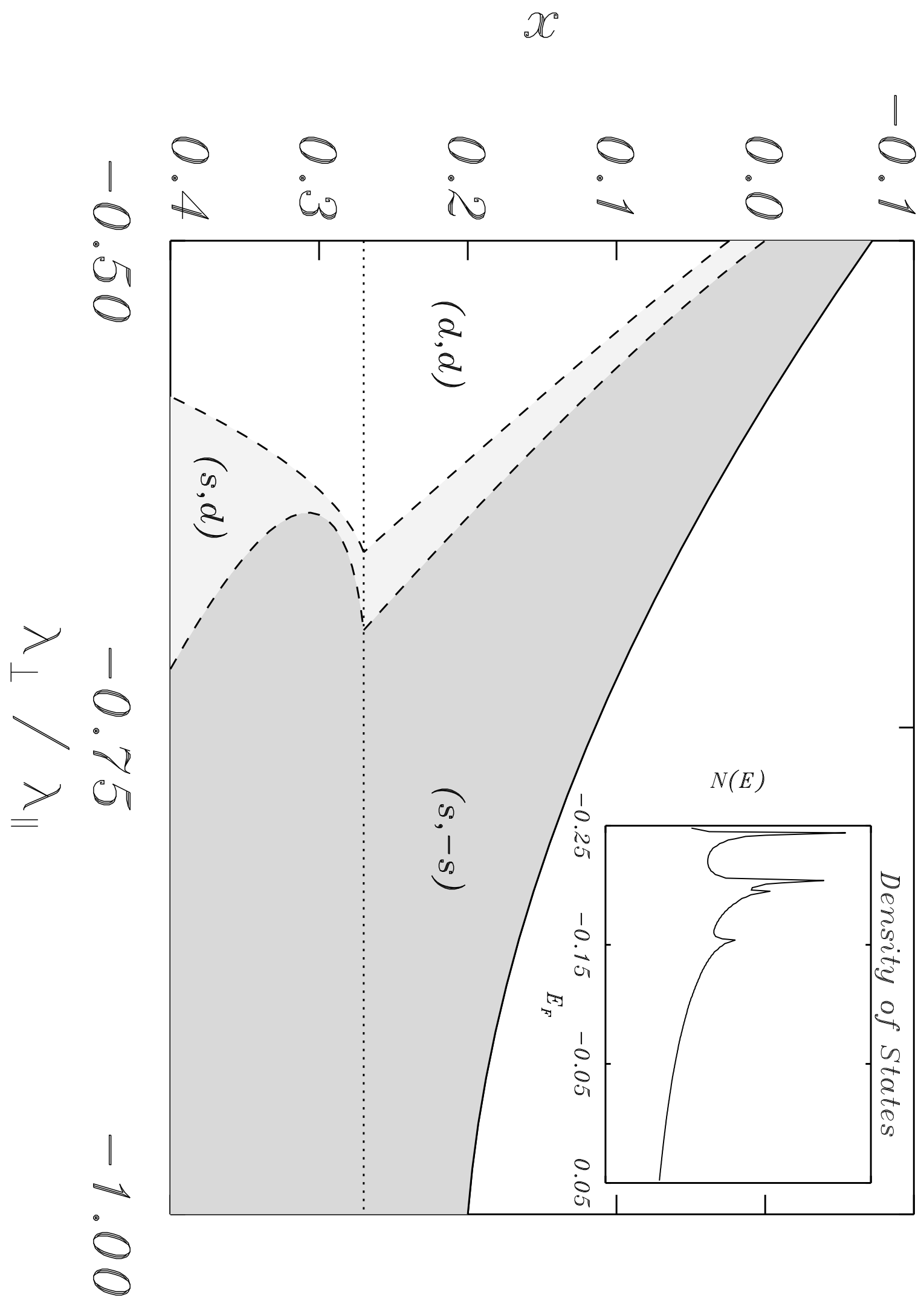


a) $\lambda_{\perp} / \lambda_{\|}=-0.50$

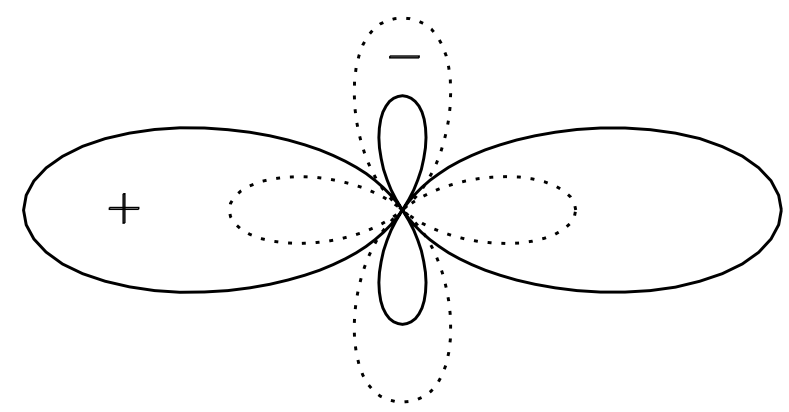

b) $\lambda_{\perp} / \lambda_{\|}=-0.68$

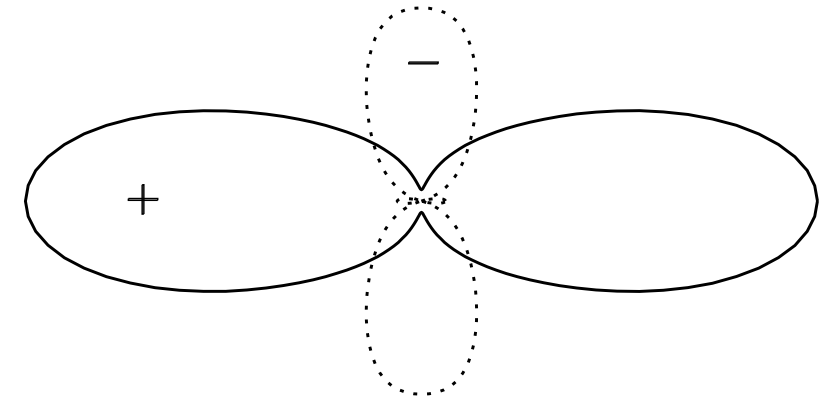

c) $\lambda_{\perp} / \lambda_{\|}=-0.80$

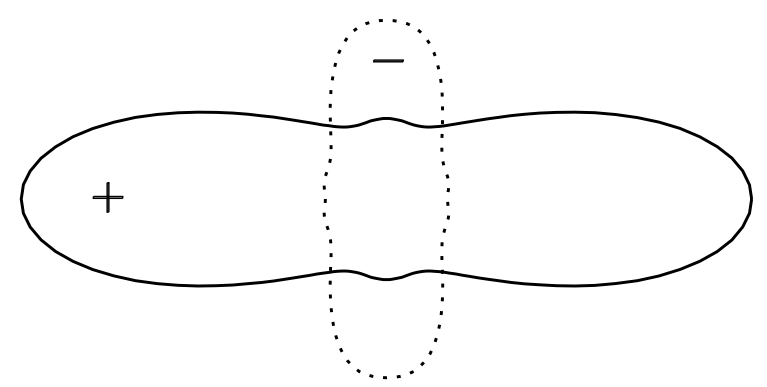

d) $\lambda_{\perp} / \lambda_{\|}=-2.00$

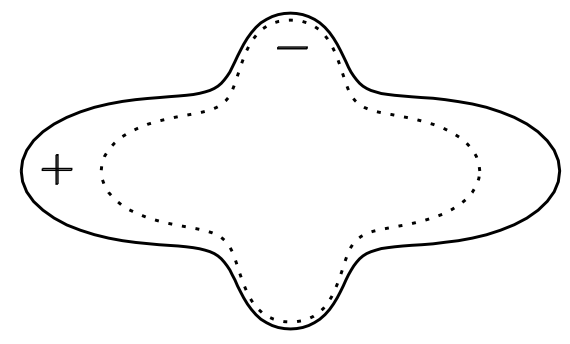




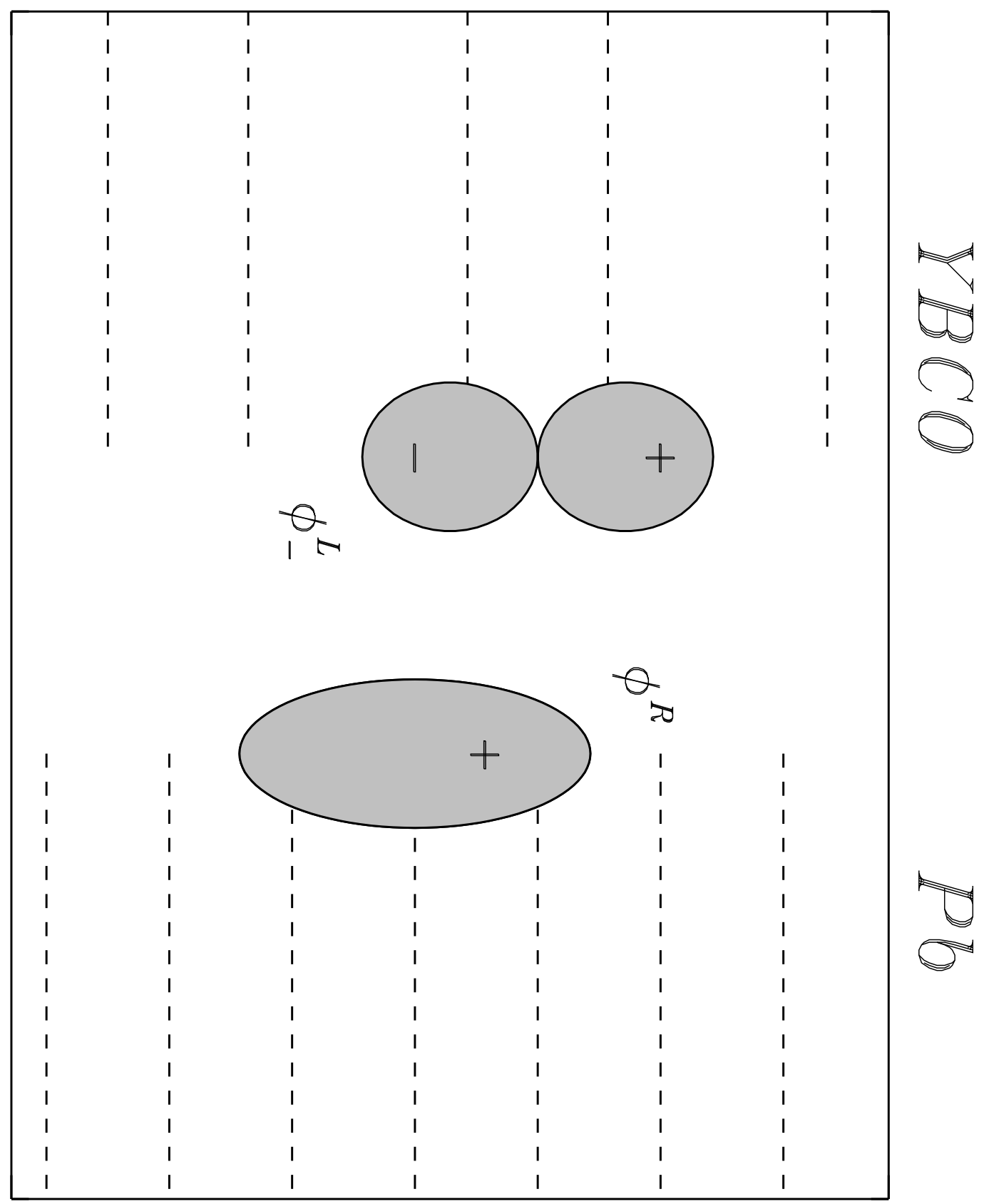




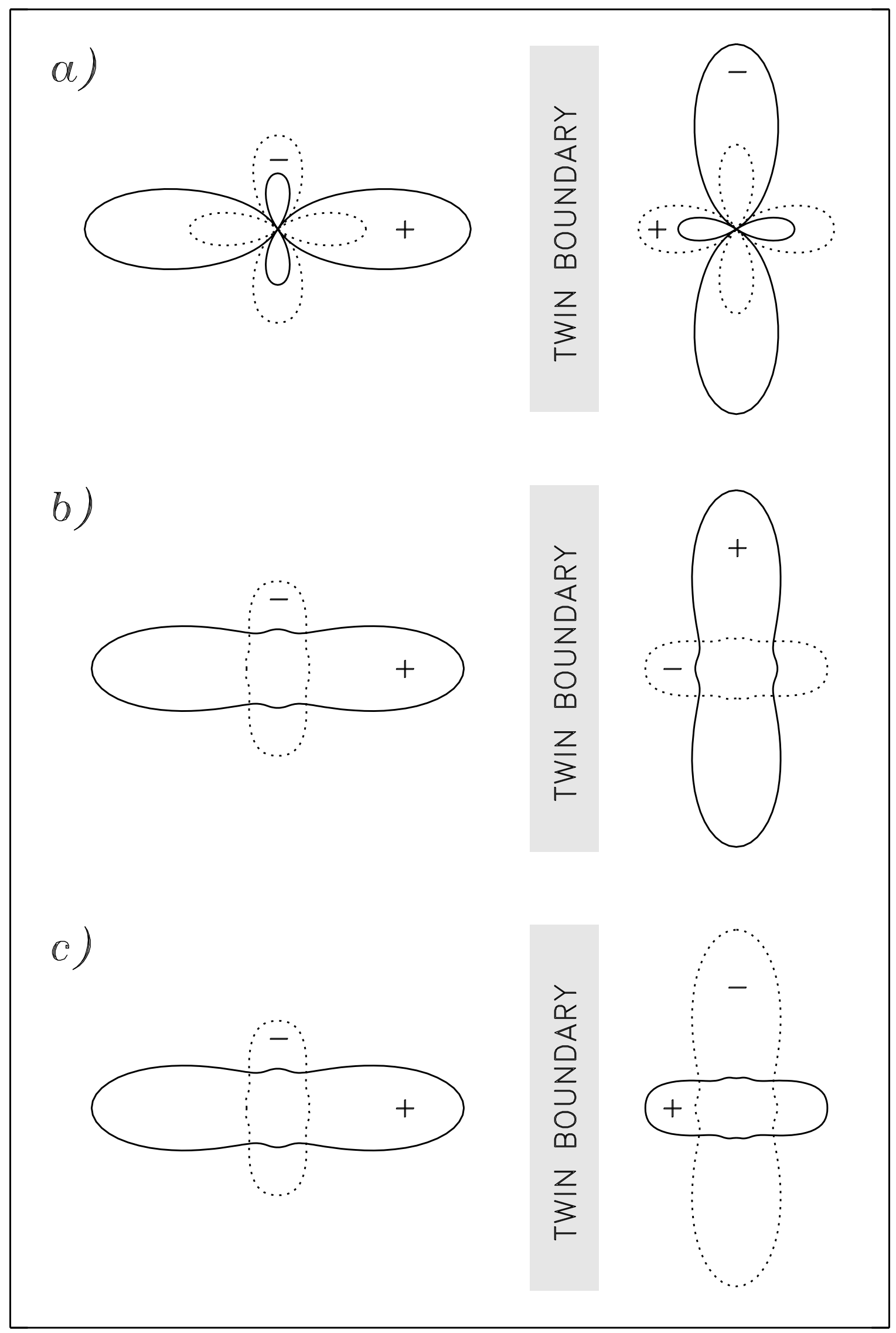




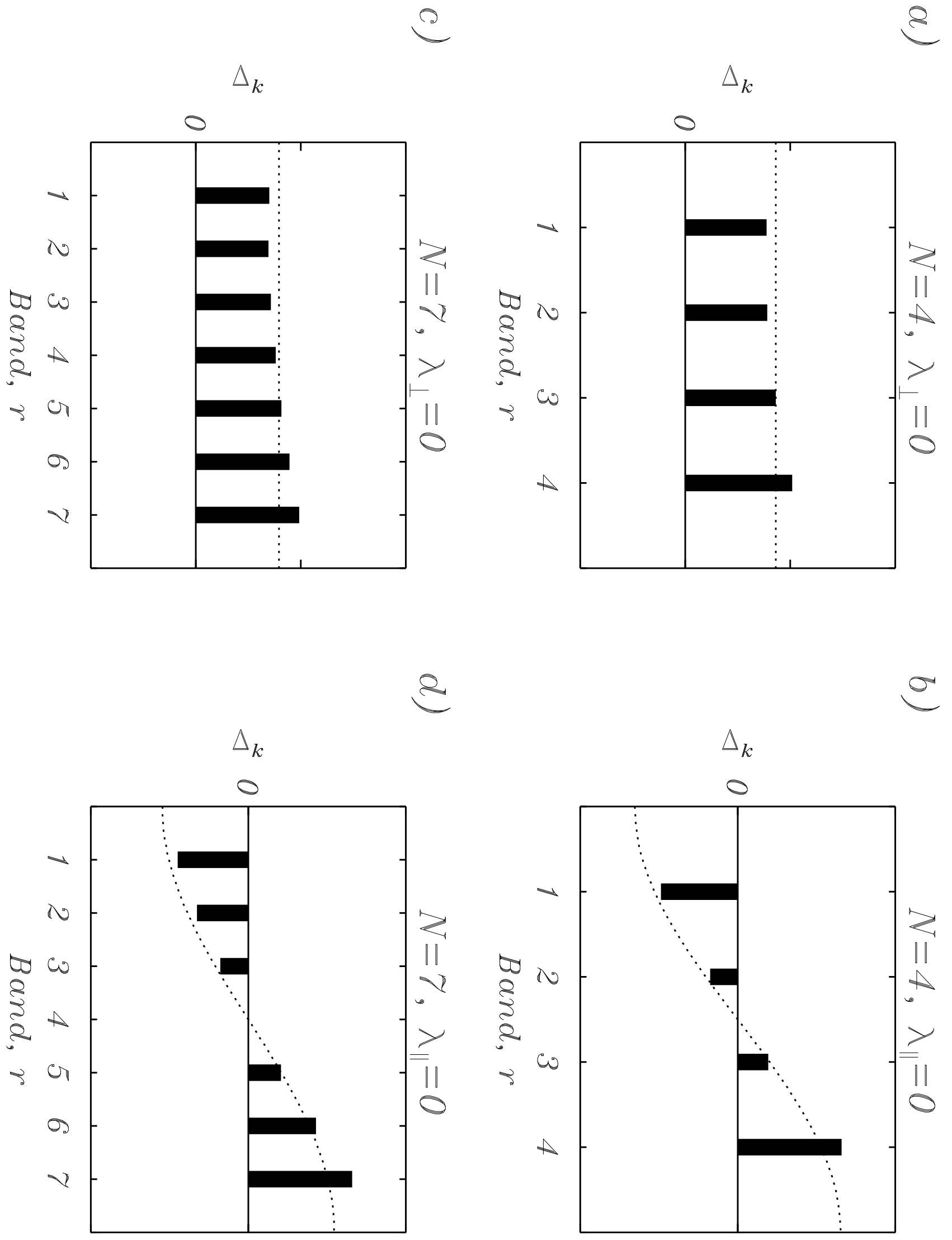




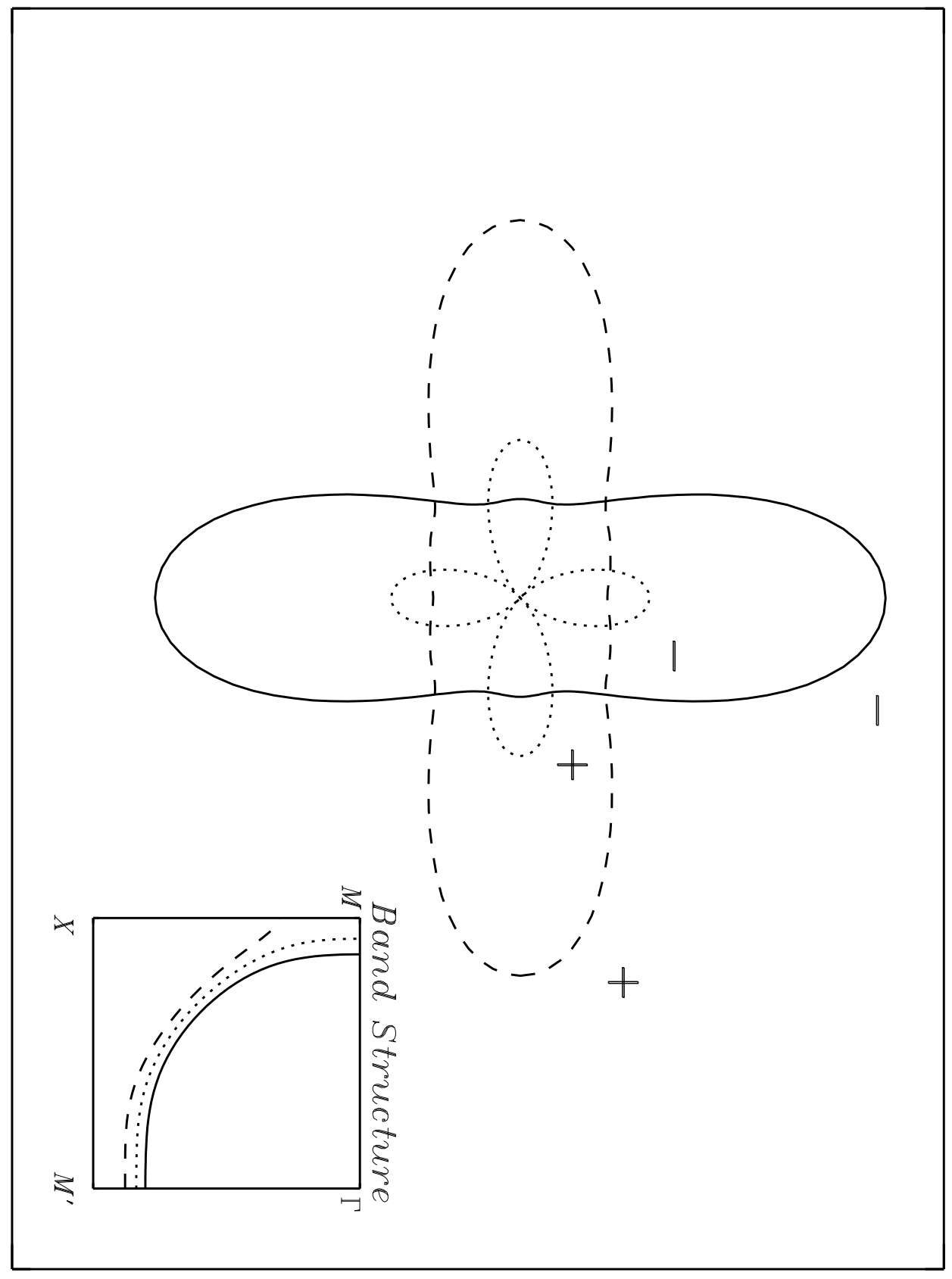


a)

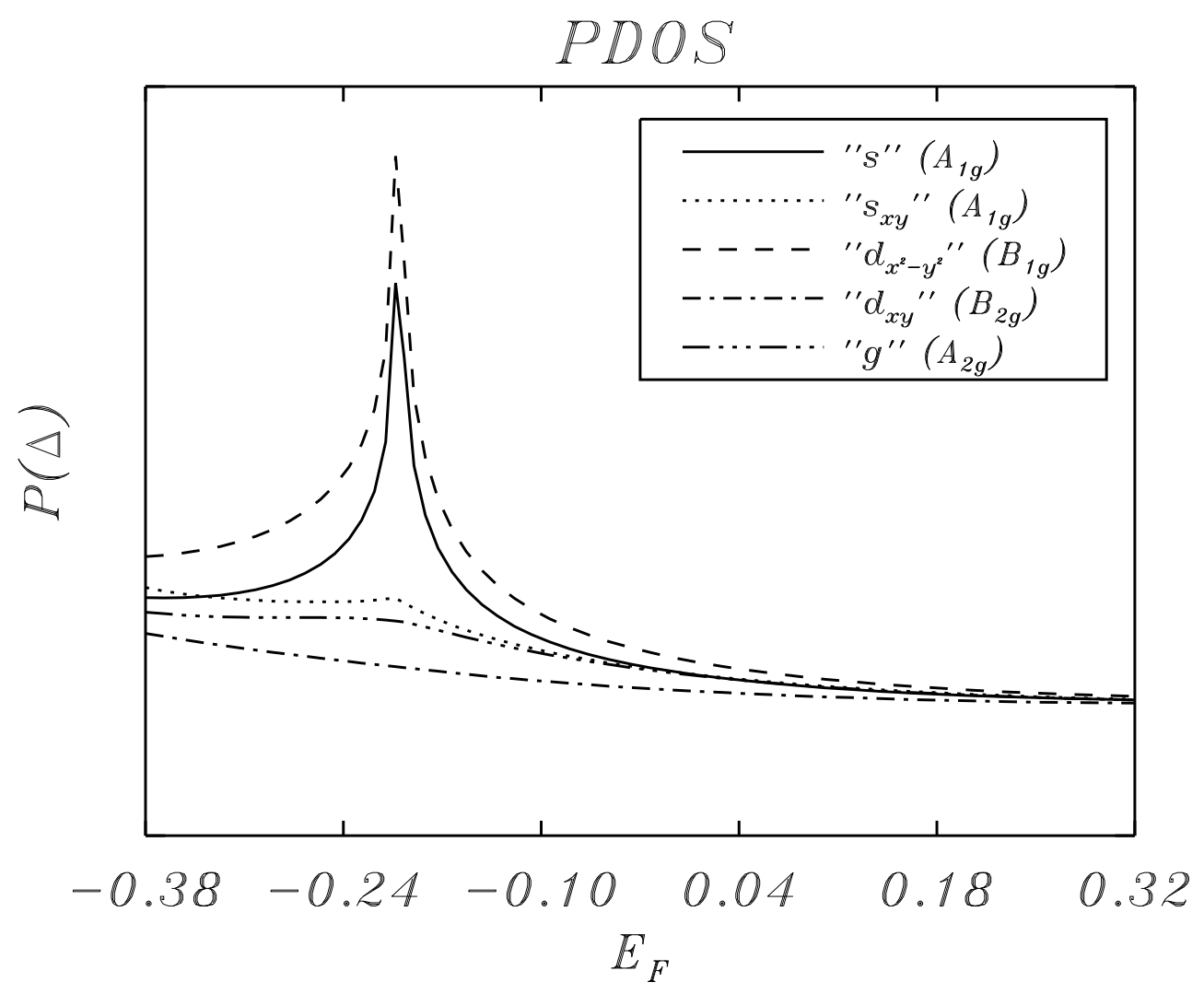

b)

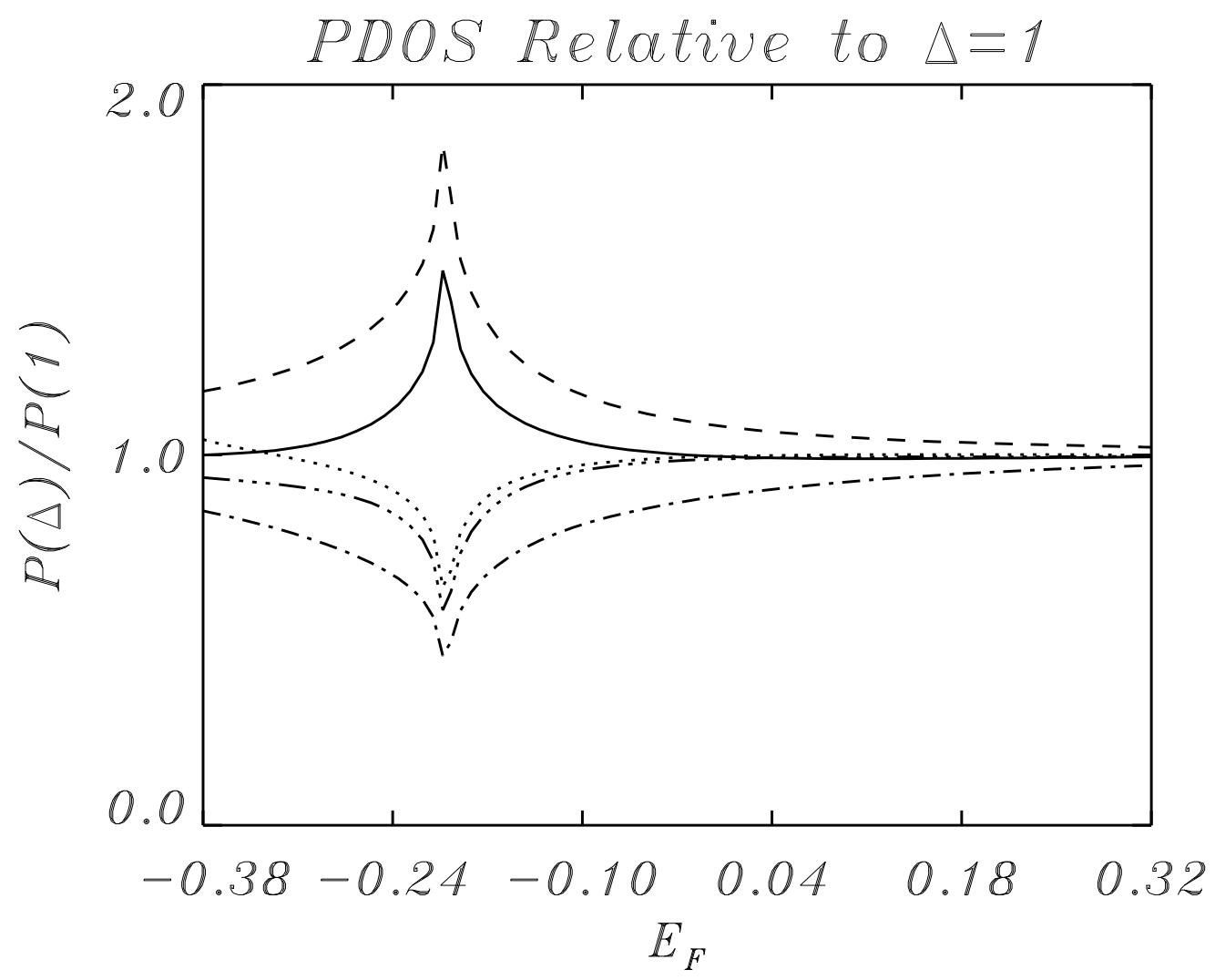

